Magazine of Concrete Research

Volume 66 Issue 6

Influence of the relative rib area on bond behaviour

Metelli and Plizzari
Magazine of Concrete Research, 2014, 66(6), 277-294

http://dx.doi.org/10.1680/macr.13.00198

Paper 1300198

Received 09/07/2013; revised 26/11/2013; accepted 27/11/2013

Published online ahead of print 24/01/2014

ICE Publishing: All rights reserved

\title{
Influence of the relative rib area on bond behaviour
}

\section{Giovanni Metelli}

Assistant Professor, Department DICATAM, University of Brescia, Brescia, Italy
Giovanni A. Plizzari

Professor, Department DICATAM, University of Brescia, Brescia, Italy

Steel-to-concrete bond is a basic aspect of the behaviour of reinforced concrete structures both at serviceability and ultimate states. When bond rules were originally developed, experimental results were mainly obtained on normalstrength concrete and a minimum relative rib area (bond index) was required by building codes to ensure good bond properties. The arrival into the market of high-performance concrete and newer structural needs may require different bond indexes. In the present paper, the experimental results of pull-out tests on short anchorages are presented. Several pull-out tests on ribbed bars, embedded in cubes of normal- and high-strength concrete with a concrete cover of 4.5 times the bar diameter, were carried out in order to better understand the influence of the relative rib area and bar diameter on the local bond behaviour, as well as on the splitting crack width generated by the wedging action of ribs. A total of 96 tests were performed on machined bars of three different diameters (12, 16 and $20 \mathrm{~mm}$ ) with a bond index ranging from 0.040 to 0.105 . The results of 55 pull-out tests on commercial hot-rolled ribbed bars of four different diameters $(12,20,40$ and $50 \mathrm{~mm})$ are also presented to confirm that the bond response also depends on bar diameter (size effect). Experimental results provide information concerning the influence of the relative rib area on bond strength and on the bursting force due to the rib's wedge action. As the minimum measured bond strength of rebars was always markedly greater than the minimum bond strength required by building codes even when low bond indexes were adopted, the test results point out the possibility of reducing the minimum value of the relative rib area required by Eurocode 2 without limiting the safety coefficient of bond. The reduction also allows a higher structural ductility that can be achieved due to a greater strain penetration of the rebars from concrete cracks.

\author{
Notation \\ $A$ nominal cross-sectional area of bar \\ $A_{\text {gt }} \quad$ elongation at maximum tensile force \\ a rib height \\ $b \quad$ rib width \\ c concrete cover \\ $d$ nominal diameter of reinforcing bar \\ $d_{\mathrm{e}} \quad$ external diameter of reinforcing bar \\ $d_{\mathrm{i}} \quad$ core diameter of reinforcing bar \\ $E_{\mathrm{cm}} \quad$ mean value of the modulus of elasticity of concrete \\ $f_{\text {bd }} \quad$ design bond strength \\ $f_{\text {bd, } 0 \quad \text { basic bond strength }}$ \\ $f_{\mathrm{cm}} \quad$ mean value of concrete compressive strength \\ (cylinder) \\ $f_{\text {cm,cube }}$ mean value of concrete compressive strength (cube) \\ $f_{\text {ctm }} \quad$ mean value of concrete tensile strength \\ $f_{\mathrm{R}} \quad$ measured bond index (or relative rib area) \\ $f_{\mathrm{Rm}} \quad$ measured mean bond index in each series \\ $f_{\mathrm{s}, 0 \cdot 2}$ \\ $f_{\text {um }}$ \\ $f_{\mathrm{yk}}$ \\ $f_{\text {ym }}$
}

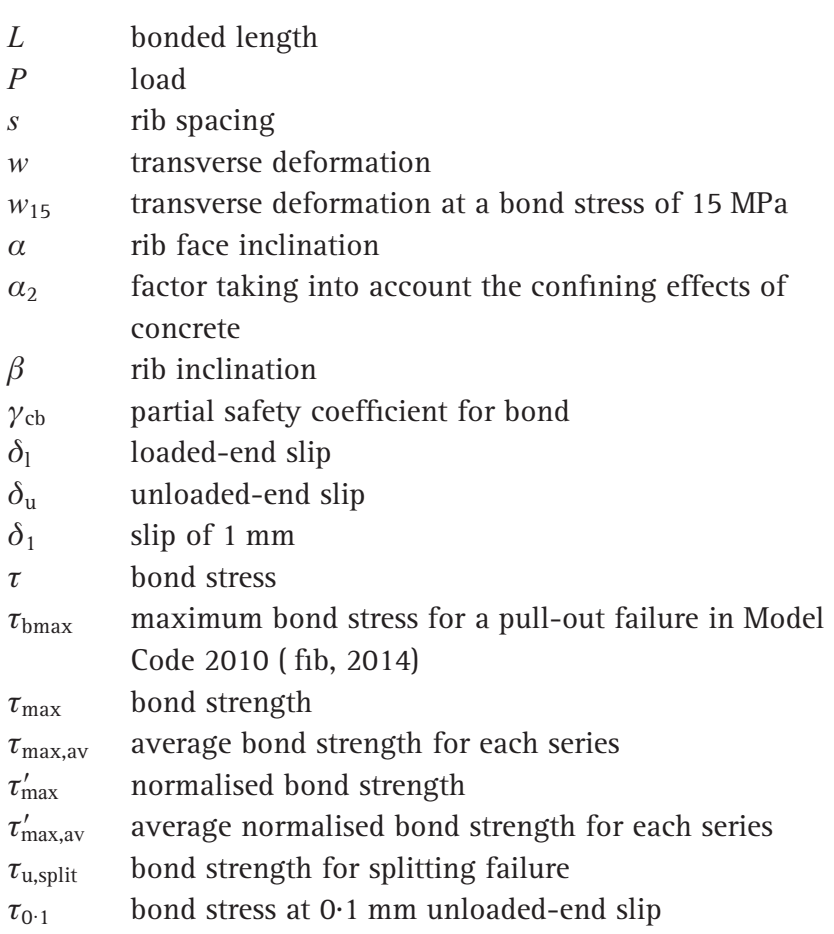




\section{Introduction}

Steel-to-concrete bond allows longitudinal forces to be transferred from the reinforcement to the surrounding concrete in a reinforced concrete $(\mathrm{RC})$ structure. Due to this stress transfer, the force in a reinforcing bar changes along its length, as does the stress in the concrete embedment. Wherever steel strains differ from concrete strains, a relative displacement between the steel and the concrete occurs (slip). Bond is commonly defined as the rate of change in force along the bar divided by the (nominal) area of bar surface over which that change takes place. However, this simple concept is quite inaccurate since the majority of bars employed today rely on the bearing of the ribs that are made to increase the bond resistance.

Due to the bursting forces on the surrounding concrete as a result of the wedge action of the ribs (Cairns and Jones, 1995a, 1995b), it has been observed that splitting cracks may develop longitudinally along the bar (Gambarova and Rosati, 1997; Giuriani et al., 1991; Tepfers, 1973). Splitting cracks impair bond mechanical behaviour and make the bond very sensitive to confinement (Plizzari et al., 1998; Tepfers, 1973). After splitting, the confining action along the anchored bars or splices is produced by transverse reinforcement (Eligehausen et al., 1983), by external transverse pressure and by cohesive stresses between the splitting crack faces (Darwin et al., 1992; Reinhardt and van der Veen, 1990; Walker et al., 1999; Xu et al., 2011). The latter confining action underlines the potential benefit of fibre reinforcement in concrete (Harajli et al., 1995; Jansson et al., 2012), even though recent studies have shown that the addition of fibres can cause a reduction in bond strength due to disturbance in the concrete matrix near the bar ribs (Dancygier and Katz, 2010).

When splitting of concrete occurs, another important aspect is structural durability. Most building codes do not have requirements on splitting crack width, whose limits should be more severe than for flexural cracks. In fact, while the latter exposes a very limited bar length to the environment, splitting cracks develop longitudinally along the bar so that a considerable length of the reinforcement can be exposed to aggressive agents (Giuriani and Plizzari, 1998).

Detailed evaluation of bond strength and bond performance is complex, as the magnitude of bond strength is influenced by a wide range of factors. As an example, Model Code 2010 (MC2010) includes about ten parameters for determining anchorage or splice strength (fib, 2013). A survey of published literature on bonding was presented by Gambarova et al. (2000).

Depending on cover and confinement, different modes of bond failure are possible - pull-out failure and splitting failure. In the former case, bond failure is mostly due to the shearing off of the concrete keys cast between each pair of lugs (Cairns and Abdullah, 1995; Giuriani, 1982). In the latter case, bond failure is mostly due to the longitudinal splitting of the concrete surrounding the bar (Cairns and Jones, 1995a).
The bond between reinforcement and concrete governs several mechanisms in RC structures, such as those related to anchorage and splice, shear behaviour and cracking. Steel-to-concrete bond should fulfil the following requirements.

(a) At service conditions, small flexural and splitting crack width and limited deflection of RC members should be ensured.

(b) At ultimate state, anchorages and lapped splice strength should be guaranteed.

(c) After yielding of the rebars, large rotation capacity at plastic hinges should be required to provide adequate ductility and energy dissipation to the structural element (Eligehausen and Mayer, 2000; Wildermuth and Hofmann, 2012).

Since these requirements are partly contradictory for bond properties, a compromise may represent an optimised solution for the rib geometry.

A review of previous studies (Cairns and Jones, 1995b; Darwin and Graham, 1993; Eligehausen and Mayer, 2000; Rhem, 1969) shows that the main rib parameter influencing bond strength and stiffness is the relative rib area, or bond index $\left(f_{R}\right)$, which is defined as the ratio between the rib area above the core, projected on a plane perpendicular to the bar axis, and the nominal bar surface area between two contiguous ribs.

When bond rules were originally developed, experimental results available in the literature were mainly obtained on normal-size rebars in normal-strength concrete (NSC). Based on these results, a minimum bond index was required by building codes to ensure good bond properties (Cairns and Plizzari, 2003). As an example, according to Eurocode 2 (CEN, 2004), a minimum bond index of 0.056 is required for a diameter larger than $12 \mathrm{~mm}$ in order to guarantee the required bond stiffness and strength.

As far as the influence of the bond index is concerned, by performing beam-end tests, Darwin and Graham (1993) showed a small increase in bond strength (10\%) when the bond index varied from 0.05 to 0.2 in bars well confined by concrete cover and transverse reinforcement; the bond-slip response was also independent of the combination of rib height and spacing. Experimental and theoretical studies on the strength of lapped joints carried out by Cairns and Jones (1995b) showed that, by doubling the bond index (from 0.05 to $0 \cdot 10$ ), the bond strength increased by $30 \%$ because of the lower bursting force generated by more highly ribbed bars.

Zuo and Darwin (2000) studied the effect of the bond index on bond-slip behaviour when reversed cyclic loading was applied; the test results showed a significant reduction of the unloadedend slip (up to 70\%) when the bond index increased from 0.085 to $0 \cdot 119$. As a result, the use of high relative rib area bars may reduce the bond damage but, at the same time, might favour strain localisation, thus limiting the rotation capacity of plastic hinges in RC structures (Eligehausen and Mayer, 2000; 
Wildermuth and Hofmann, 2012). Numerical simulations carried out by Wildermuth and Hofmann (2012) showed an increase in the plastic rotation of a beam by more than $50 \%$ when the bond index decreased from 0.09 to 0.02 and by $20 \%$ when the bond index decreased from 0.09 to 0.07 .

There is therefore a need to optimise the relative rib area in order to obtain a good bond strength for anchorages as well as an enhanced plastic hinge (possible with lower bond strength and stiffness) for structural ductility. Furthermore, the arrival into the market of high-performance concrete and newer structural needs may require different bond indexes.

The aim of the present research work was to investigate the influence of rib geometry on the bond strength and stiffness, as well as on the splitting cracks due to the bursting forces generated by the wedge action of the ribs. Several pull-out tests were performed on rebars with different diameters and rib geometries. Furthermore, the size effect on bond behaviour was also studied by performing pull-out tests on large rebars of diameter $40 \mathrm{~mm}$ and $50 \mathrm{~mm}$.

\section{Experimental work and materials}

Twelve series of pull-out tests were performed on concrete specimens (cubes) with an embedded bar having a nominal diameter $(d)$ of 12,16 or $20 \mathrm{~mm}$. The bar diameters were chosen in order to obtain an approximately constant ratio between the bonded surfaces of two contiguous diameters $\left(A_{20} / A_{16}=1 \cdot 56\right.$; $\left.A_{16} / A_{12}=1 \cdot 78\right)$.

For each bar diameter, two values of the bond index $\left(f_{\mathrm{R}}\right)$ were investigated - a lower bond index value, close to $0 \cdot 065$, and an upper value, close to 0.095 , for $16 \mathrm{~mm}$ and $20 \mathrm{~mm}$ diameter bars; the $12 \mathrm{~mm}$ bars (with a lower minimum requirement by codes) had a bond index ranging between 0.040 (lower) and 0.096 (upper).

Smooth bars were machined in order to obtain helical ribs adopting a commonly used trapezoidal profile with an inclination $(\beta)$ of roughly $80^{\circ}$ to the bar axis and a rib face inclination $(\alpha)$ of $50^{\circ}$ (Figure 1). The rib spacing $(s)$ varied between $6.0 \mathrm{~mm}$ and $13.9 \mathrm{~mm}$ in the $12 \mathrm{~mm}$ and $20 \mathrm{~mm}$ bars respectively. The conventional value of the ratio between the nominal diameter $(d)$ and the rib height (a) was constant and equal to 22, with the exception of the $12 \mathrm{~mm}$ bars (with $f_{\mathrm{Rm}}=0 \cdot 047$ ), where the ratio was 32. The measurement of rib height $(a)$ and external bar diameter $\left(d_{\mathrm{e}}\right)$ were taken at $120^{\circ}$ intervals along the helical ribs and averaged. For each bar, the bond index was calculated using

1. $f_{\mathrm{R}}=\frac{d_{\mathrm{e}}^{2}-d_{\mathrm{i}}^{2}}{4 d s}$

2. $d_{\mathrm{i}}=d_{\mathrm{e}}-2 a$
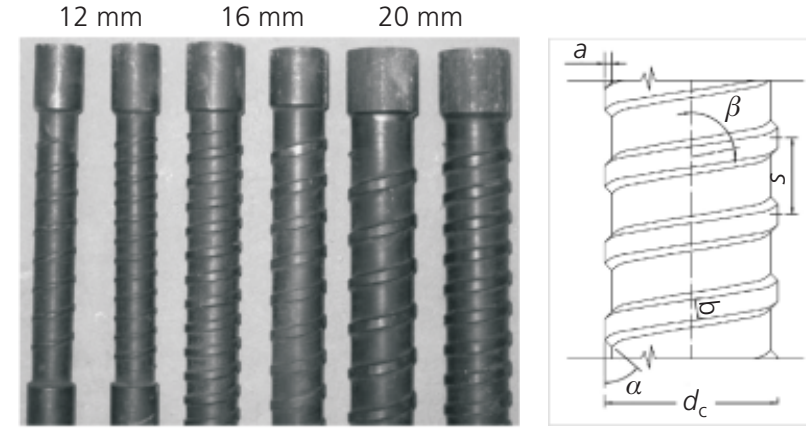

(a)

$\begin{array}{llll}d=40 \mathrm{~mm} & d=40 \mathrm{~mm} & d=50 \mathrm{~mm} & d=50 \mathrm{~mm} \\ f_{\mathrm{Rm}}=0.054 & f_{\mathrm{Rm}}=0.072 & f_{\mathrm{Rm}}=0.040 & f_{\mathrm{Rm}}=0.063\end{array}$

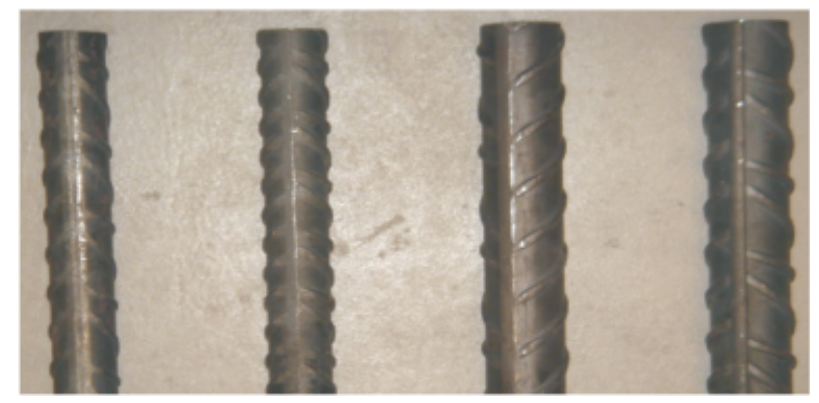

(b)

Figure 1. (a) Machined ribbed bars and geometry. (b) Hot-rolled commercial bars

in which $d_{\mathrm{e}}$ is the external bar diameter (top of the rib), $d_{\mathrm{i}}$ is the core diameter (bottom of the rib), $d$ is the nominal diameter, $s$ is longitudinal spacing of the ribs and $a$ is the rib height. The geometric properties of the machined steel bars are shown in Figure 1(a) and listed in Table 1 (also see the Appendix).

High-grade steel was used with a mean yield strength $\left(f_{\mathrm{ym}}\right)$ of $940 \mathrm{MPa}$ in order to prevent the bar yielding before bond failure (Table 2). As each series consisted of eight specimens and the bars were tested both in normal- and high-strength concrete (NSC and HSC), 96 specimens with an embedded machined bar were tested.

A further eight series of pull-out tests, consisting of five specimens each (at least), were performed on commercial hot-rolled bars of diameter 12, 20, 40 and $50 \mathrm{~mm}$. The 12 and $20 \mathrm{~mm}$ ribbed bars were characterised by a bond index greater than the minimum required by Eurocode 2 and they were tested both in NSC and HSC; the 40 and $50 \mathrm{~mm}$ diameter bars were tested only in NSC. For each large-diameter bar, two values of the bond index were investigated (Figure 1(b))

- lower than the minimum value required by Eurocode 2 $\left(f_{\mathrm{Rm}}=0.040\right.$ or $f_{\mathrm{Rm}}=0.054$ respectively for 50 and $40 \mathrm{~mm}$ bar diameters)

- greater than the minimum value required by Eurocode 2 


\begin{tabular}{lccccccc}
\hline$d: m m$ & $f_{\mathrm{Rm}}$ & $d_{\mathrm{e}}: \mathrm{mm}$ & $\begin{array}{c}\beta: \\
\text { degrees }\end{array}$ & $s: \mathrm{mm}$ & $a: \mathrm{mm}$ & $b: \mathrm{mm}$ & $\begin{array}{c}\alpha: \\
\text { degrees }\end{array}$ \\
\hline 12 & 0.047 & 13 & 77.90 & 8.50 & 0.38 & 1.5 & 50 \\
12 & 0.081 & 13 & 81.65 & 6.00 & 0.55 & 1.5 & 50 \\
16 & 0.061 & 17 & 78.40 & 10.95 & 0.70 & 2.0 & 50 \\
16 & 0.095 & 17 & 82.00 & 7.50 & 0.70 & 2.0 & 50 \\
20 & 0.065 & 21 & 78.10 & 13.90 & 0.90 & 2.5 & 50 \\
20 & 0.092 & 21 & 81.80 & 9.50 & 0.90 & 2.5 & 50
\end{tabular}

Table 1. Geometric properties of the machined reinforcing bars (nominal values)

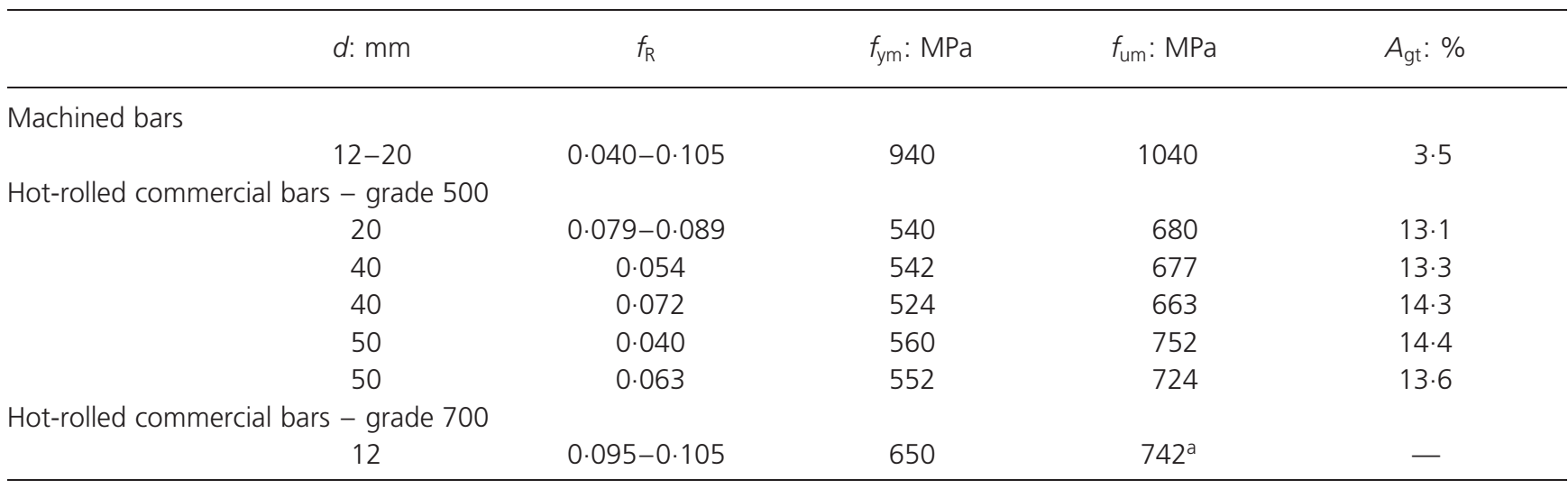

a Note this value is for $f_{5,0 \cdot 2}$ (in $\mathrm{MPa}$ ).

Table 2. Mechanical properties of the reinforcing bars

$\left(f_{\mathrm{Rm}}=0.063\right.$ or $f_{\mathrm{Rm}}=0.072$ respectively for 50 and $40 \mathrm{~mm}$ bar diameters).

The large bars were tested only in NSC. The relative rib area of each hot-rolled bar was measured according to ISO 15630-1 (ISO, 2010). A normal steel was used for 20, 40 and $50 \mathrm{~mm}$ diameter bars, with a mean yield strength $f_{\mathrm{ym}}=524-560 \mathrm{MPa}$ (Table 2). The $12 \mathrm{~mm}$ diameter bars were made of steel with a higher strength $\left(f_{\mathrm{ym}}=704 \mathrm{MPa}\right)$.

The concrete was poured into wooden forms with the bars in a horizontal position in order to obtain homogeneous bond conditions along the anchorage length. Specimens were cast in groups of nine (three series, each consisting of three specimens) from a single batch of concrete. The concrete mix proportions are shown in Table 3; the aggregates for the mix were defined by weight according to the percentages shown in Table 4 .

The cast specimens were left for $36 \mathrm{~h}$ and then demoulded and stored in a humidity room at constant $90 \%$ humidity and temperature $20^{\circ} \mathrm{C}$ until the time of the test. From each batch, four cylindrical samples ( $80 \mathrm{~mm}$ diameter, $160 \mathrm{~mm}$ high) and

\begin{tabular}{llccccc}
\hline$d: m m$ & Concrete & Cement: $\mathrm{kg} / \mathrm{m}^{3}$ & Water: $1 / \mathrm{m}^{3}$ & W/c & Aggregate: $\mathrm{kg} / \mathrm{m}^{3}$ & Superplasticiser: $1 / \mathrm{m}^{3}$ \\
\hline 40,50 & NSC & $325($ R325) & 160 & $0 \cdot 49$ & 1949 & $3 \cdot 0$ \\
$12,16,20$ & NSC & $350($ R425) & 180 & $0 \cdot 51$ & 1815 & $3 \cdot 5$ \\
$12,16,20$ & HSC & $450($ R525) & 142 & $0 \cdot 32$ & 1885 & $5 \cdot 0$
\end{tabular}

Table 3. Composition of normal-strength and high-strength concretes 


\begin{tabular}{|c|c|c|}
\hline \multirow[t]{2}{*}{ Grain class: mm } & \multicolumn{2}{|c|}{ Percentage: \% } \\
\hline & NSC & $\mathrm{HSC}$ \\
\hline $0.00-0.35$ & $10 \cdot 56$ & $7 \cdot 12$ \\
\hline $0.35-0.45$ & $12 \cdot 72$ & $9 \cdot 36$ \\
\hline $0.40-0.60$ & $15 \cdot 55$ & $12 \cdot 30$ \\
\hline $0.60-1.50$ & $27 \cdot 82$ & 25.04 \\
\hline $1 \cdot 50-2 \cdot 50$ & 37.53 & $35 \cdot 13$ \\
\hline $2 \cdot 50-3 \cdot 50$ & 48.95 & $46 \cdot 98$ \\
\hline $4 \cdot 00-6 \cdot 00$ & $61 \cdot 20$ & $59 \cdot 71$ \\
\hline $7 \cdot 00-12 \cdot 00$ & $80 \cdot 63$ & $79 \cdot 88$ \\
\hline $10 \cdot 00-15.00$ & $100 \cdot 00$ & $100 \cdot 00$ \\
\hline
\end{tabular}

Table 4. Aggregate composition in normal-strength and highstrength concretes

eight cubes (side $150 \mathrm{~mm}$ ) were cast and cured in the same conditions as the pull-out specimens. At the time of testing, the NSC specimens presented a compressive cubic strength $f_{\text {cm,cube }}=42 \cdot 7-47 \cdot 8 \mathrm{MPa}$, while the compressive strength of the HSC specimens ranged between $63.4 \mathrm{MPa}$ and $74.3 \mathrm{MPa}$ (average values). The curing time and the mechanical characteristics of the concrete specimens are summarised in Table 5 .

For the specimens with a large bar diameter, the concrete was poured into wooden forms with the bars in a horizontal position. The 24 specimens were demoulded $24 \mathrm{~h}$ after casting and were then stored in the laboratory at a temperature of about $22^{\circ} \mathrm{C}$ and relative humidity about $45 \%$. As shown in Table 5, at the time of testing, the concrete compressive strength $\left(f_{\mathrm{cm}, \text { cube }}\right)$ of the specimens with large bars was $37.6 \mathrm{MPa}$.

\section{Test set-up}

The test equipment (Figure 2) used was as for a typical pull-out test proposed by Rilem/CEB/FIP (1978). The specimen was characterised by an embedded length of five bar diameters in a concrete cube having a side of ten bar diameters. A plastic sleeve rendered the rebar unbonded over half the depth of the cubic specimen (five bar diameters) and a $2 \mathrm{~mm}$ thick Teflon sheet reduced friction at the bearing surface of the specimen.

During the test, the unloaded-end slip $\left(\delta_{\mathrm{u}}\right)$ was measured by a linear variable differential transformer (LVDT) centrally mounted on the bar while the loaded-end slip $\left(\delta_{1}\right)$ was measured by two

\begin{tabular}{|c|c|c|c|c|c|c|}
\hline Series & $d: m m$ & $f_{\mathrm{Rm}}$ & $\begin{array}{l}\text { Curing } \\
\text { time: days }\end{array}$ & $\begin{array}{c}f_{\mathrm{cm}, \text { cube }}: \\
\mathrm{MPa}\end{array}$ & $\begin{array}{l}f_{\mathrm{ctm}}: \\
\mathrm{MPa}\end{array}$ & $\begin{array}{l}E_{\mathrm{cm}}: \\
\mathrm{MPa}\end{array}$ \\
\hline 1 & 12 & 0.047 & 34 & $42 \cdot 72$ & $3 \cdot 4$ & 26672 \\
\hline 2 & 16 & 0.061 & & & & \\
\hline 3 & 20 & 0.065 & & & & \\
\hline 4 & 12 & 0.081 & 29 & $47 \cdot 82$ & $4 \cdot 3$ & 28059 \\
\hline 5 & 16 & 0.095 & & & & \\
\hline 6 & 20 & 0.092 & & & & \\
\hline 7 & 12 & 0.047 & 28 & $63 \cdot 40$ & $4 \cdot 3$ & 31024 \\
\hline 8 & 16 & 0.061 & & & & \\
\hline 9 & 20 & 0.065 & & & & \\
\hline 10 & 12 & 0.081 & 21 & $74 \cdot 33$ & $4 \cdot 5$ & 30215 \\
\hline 11 & 16 & 0.095 & & & & \\
\hline 12 & 20 & 0.092 & & & & \\
\hline 13 & 12 & 0.095 & 38 & $41 \cdot 50$ & $3 \cdot 4$ & 29733 \\
\hline 14 & 20 & 0.089 & & & & \\
\hline 15 & 12 & 0.089 & 90 & 64.80 & $3 \cdot 2$ & 33270 \\
\hline 16 & 20 & 0.079 & & & & \\
\hline 17 & 40 & 0.054 & 40 & 37.60 & 3.0 & 26500 \\
\hline 18 & 40 & 0.072 & & & & \\
\hline 19 & 50 & 0.040 & & & & \\
\hline 20 & 50 & 0.063 & & & & \\
\hline
\end{tabular}

$f_{\mathrm{Rm}}=$ average value of the actual bond index; $f_{\mathrm{cm}, \mathrm{cube}}=$ mean compressive strength from eight cubes; $f_{\mathrm{ctm}}=$ mean tensile strength from two cylinders; $E_{\mathrm{cm}}=$ mean tangent modulus of elasticity from two cylinders.

Table 5. Mechanical properties of the concrete specimens 


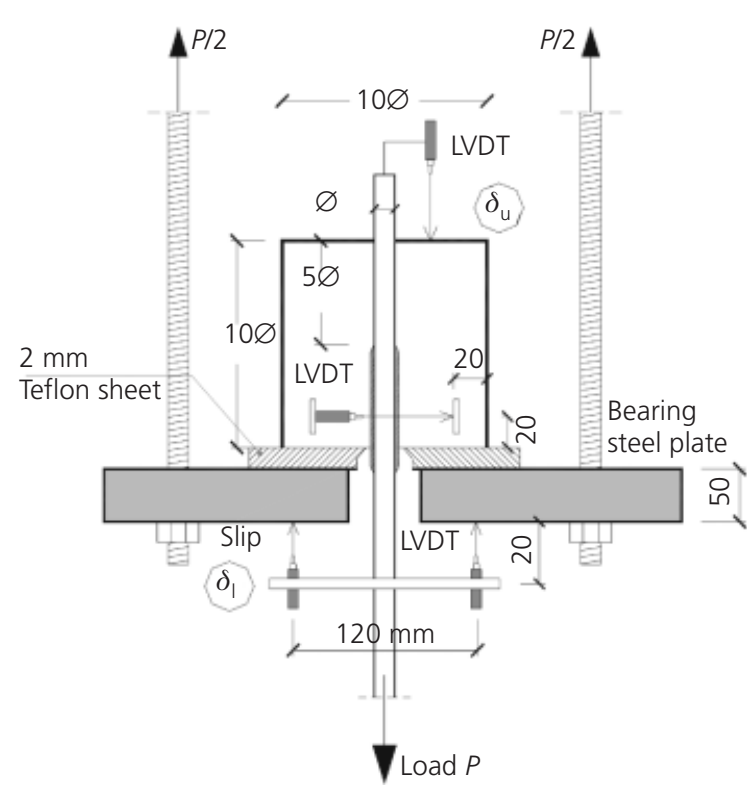

(a)

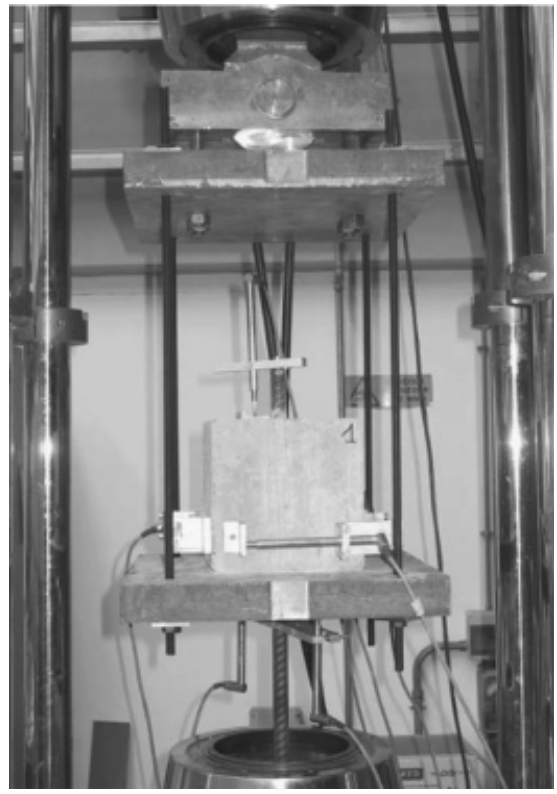

(b)

Figure 2. (a) Test set-up for normal-size bars. (b) A specimen and instrumentation

LVDTs placed at $180^{\circ}$, as shown in Figure 2. An LVDT placed on each side face of the specimen, at a distance of $20 \mathrm{~mm}$ from the Teflon-concrete interface, provided the transverse deformation $w$ (including the splitting crack, where present). The latter measurement can provide information on the deformation or on the splitting crack due to bursting forces generated by the bond action.

The tests were displacement controlled up to failure with a loaded-end displacement rate of $0 \cdot 1 \mathrm{~mm} / \mathrm{min}$. The displacement rate was calibrated in order to obtain accurate data of the ascending branch of the bond-slip relationship, with a single test lasting about $2 \mathrm{~h}$.

Due to their dimensions, the specimens with large-diameter bars

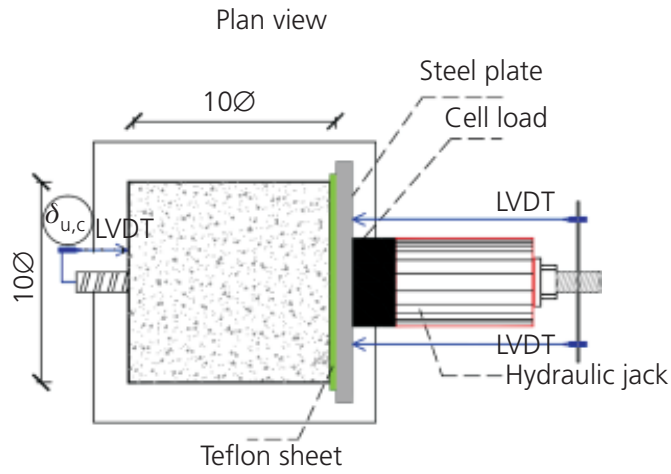

were tested horizontally, with a hydraulic jack to apply the load (Figure 3 ). The tests were load controlled with a load rate of 0.9 and $1.4 \mathrm{kN} / \mathrm{s}$ for the 40 and $50 \mathrm{~mm}$ bar diameters respectively. As for the normal-size bars, the unloaded-end slip and the loadedend slip were measured. Further details can be found in the literature (Metelli et al., 2010).

\section{Test results and discussion}

The experimental results concern the bond stress $\tau$, the unloadedend slip $\delta_{\mathrm{u}}$ and the transverse deformation $w$ for the 151 specimens, characterised by five diameters, two concrete grades and a bond index in the range 0.04 to $0 \cdot 105$. Mean values from a single series of hot-rolled commercial bars were generally considered and compared to the results of specimens with the machined bar.

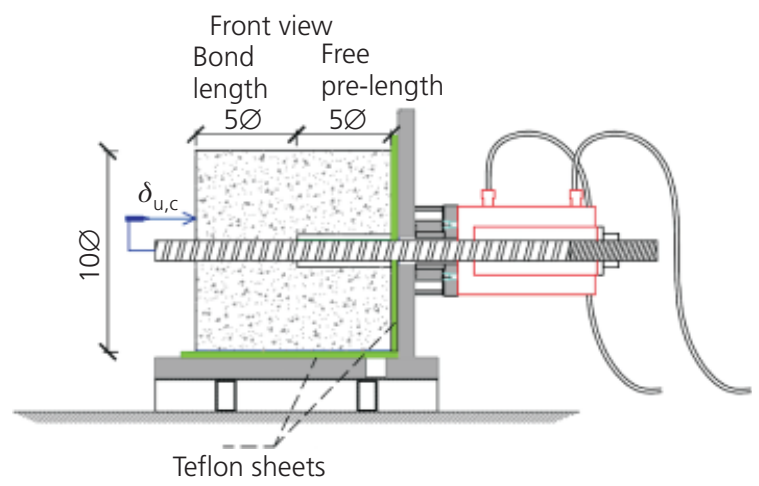

Figure 3. Test set-up for large-diameter bars 
With regard to the machined bars, all 48 HSC specimens were tested successfully. Two tests in NSC, concerning a $12 \mathrm{~mm}$ and a $20 \mathrm{~mm}$ bar, were discarded because the testing machine could not control the tests accurately. Furthermore, five specimens with results having a relative deviation greater than $30 \%$ within the same series were neglected.

Since a short anchorage length was used, the bond stress $\tau$ is conventionally assumed to be uniformly distributed along the embedded surface of the rebar and it is evaluated by

3. $\tau=P / \pi d L$

where $P$ is the applied load, $d$ is the nominal bar diameter and $L$ is the nominal bonded length (equal to $5 d$ ).

The main experimental results are listed in Table 6 for the machined bars and Table 7 for hot-rolled commercial bars. As far as the machined bars are concerned, most of the specimens failed because of the development of a splitting crack on a longitudinal plane including the bar axis (Figure 4); the only exceptions were found in seven HSC specimens with a rebar having a bond index of about 0.065 (three $16 \mathrm{~mm}$ machined bars and four $20 \mathrm{~mm}$ machined bars), in one NSC specimen with a $12 \mathrm{~mm}$ machined bar (having a bond index of 0.075) and in six specimens with commercial bars that suffered pull-out failure.

Comparison of the bond stress $(\tau)$ versus unloaded-end slip $\left(\delta_{\mathrm{u}}\right)$ curves between series with different bond indexes but the same diameter and concrete strength are shown in Figure 5. Figure 6 shows the same comparison between series with a different concrete strength but the same bond index and bar diameter. Each series, consisting of eight specimens of normalsize diameter $(12,16$ and $20 \mathrm{~mm})$ and at least five specimens of large-diameter ( 40 and $50 \mathrm{~mm}$ ) bars, is labelled by the average value of the measured bond index $f_{\mathrm{Rm}}$. The curves show better behaviour of the specimens with a greater bond index or concrete strength, both in terms of bond strength and stiffness.

\begin{tabular}{|c|c|c|c|c|c|c|c|c|}
\hline Specimen & $f_{\mathrm{R}}$ & $P_{\max }: \mathrm{kN}$ & $\tau_{\max }: \mathrm{MPa}$ & $\tau_{0 \cdot 1}: \mathrm{MPa}$ & $W_{15}: \mathrm{mm}$ & Failure mode & $\begin{array}{c}\tau_{\text {max av }}: \\
M P a\end{array}$ & $\begin{array}{c}\tau_{\max , a v} / f_{\mathrm{bd}} \\
(\mathrm{MC} 2010)\end{array}$ \\
\hline \multicolumn{9}{|c|}{ Series $1, f_{\mathrm{Rm}}=0.047, f_{\mathrm{cm}}=42.7 \mathrm{MPa}, d=12 \mathrm{~mm}$} \\
\hline 1 & 0.043 & - & - & $11 \cdot 52$ & - & - & \multirow[t]{8}{*}{$25 \cdot 7$} & \multirow[t]{8}{*}{$7 \cdot 99$} \\
\hline 2 & 0.058 & $59 \cdot 7$ & $26 \cdot 38$ & $13 \cdot 63$ & 0.013 & SPL & & \\
\hline 3 & 0.042 & $62 \cdot 6$ & $27 \cdot 69$ & $15 \cdot 43$ & 0.011 & SPL & & \\
\hline 4 & 0.057 & $60 \cdot 5$ & $26 \cdot 76$ & $16 \cdot 53$ & $0 \cdot 015$ & SPL & & \\
\hline $5^{a}$ & 0.040 & $37 \cdot 5$ & $16 \cdot 57$ & $15 \cdot 84$ & 0.024 & SPL & & \\
\hline 6 & 0.052 & $45 \cdot 3$ & $20 \cdot 02$ & $16 \cdot 07$ & $0 \cdot 010$ & SPL & & \\
\hline 7 & 0.043 & $60 \cdot 5$ & $26 \cdot 74$ & $12 \cdot 95$ & 0.020 & SPL & & \\
\hline 8 & 0.040 & $60 \cdot 6$ & $26 \cdot 81$ & $13 \cdot 46$ & 0.012 & SPL & & \\
\hline \multicolumn{9}{|c|}{ Series $2, f_{\mathrm{Rm}}=0.061, f_{\mathrm{cm}}=42.7 \mathrm{MPa}, d=16 \mathrm{~mm}$} \\
\hline 9 & 0.058 & $92 \cdot 0$ & $22 \cdot 88$ & $6 \cdot 00$ & 0.030 & SPL & \multirow[t]{8}{*}{$22 \cdot 9$} & \multirow[t]{8}{*}{$7 \cdot 11$} \\
\hline 10 & 0.065 & 108.7 & 27.04 & 6.05 & 0.008 & $\mathrm{SPL}$ & & \\
\hline 11 & 0.056 & $86 \cdot 7$ & $21 \cdot 56$ & 7.95 & 0.016 & SPL & & \\
\hline 12 & 0.061 & 99.5 & $24 \cdot 75$ & $11 \cdot 64$ & 0.022 & SPL & & \\
\hline $13^{a}$ & 0.059 & $61 \cdot 4$ & $15 \cdot 28$ & 7.95 & 0.030 & $\mathrm{SPL}$ & & \\
\hline 14 & 0.063 & 79.9 & $19 \cdot 86$ & $9 \cdot 85$ & 0.023 & $\mathrm{SPL}$ & & \\
\hline 15 & 0.058 & $78 \cdot 8$ & $19 \cdot 58$ & $12 \cdot 64$ & 0.027 & SPL & & \\
\hline 16 & 0.066 & 98.8 & $24 \cdot 57$ & $8 \cdot 40$ & 0.017 & $\mathrm{SPL}$ & & \\
\hline \multicolumn{9}{|c|}{ Series $3, f_{\mathrm{Rm}}=0.065, f_{\mathrm{cm}}=42.7 \mathrm{MPa}, d=20 \mathrm{~mm}$} \\
\hline 17 & 0.063 & $120 \cdot 1$ & $19 \cdot 11$ & - & - & SPL & \multirow[t]{8}{*}{$20 \cdot 9$} & \multirow[t]{8}{*}{$6 \cdot 49$} \\
\hline 18 & 0.073 & $144 \cdot 8$ & $23 \cdot 04$ & $5 \cdot 22$ & 0.020 & SPL & & \\
\hline 19 & 0.068 & $141 \cdot 9$ & $22 \cdot 58$ & $5 \cdot 20$ & 0.021 & SPL & & \\
\hline 20 & 0.049 & $147 \cdot 3$ & $23 \cdot 45$ & $6 \cdot 19$ & 0.027 & SPL & & \\
\hline 21 & 0.066 & $128 \cdot 1$ & $20 \cdot 39$ & $8 \cdot 14$ & 0.023 & SPL & & \\
\hline 22 & 0.069 & $129 \cdot 0$ & $20 \cdot 53$ & $6 \cdot 23$ & 0.031 & SPL & & \\
\hline $23^{a}$ & 0.063 & 84.7 & $13 \cdot 48$ & $13 \cdot 24$ & - & SPL & & \\
\hline 24 & 0.069 & $108 \cdot 5$ & $17 \cdot 26$ & $8 \cdot 14$ & 0.015 & SPL & & \\
\hline
\end{tabular}




\begin{tabular}{|c|c|c|c|c|c|c|c|c|}
\hline Specimen & $f_{\mathrm{R}}$ & $P_{\text {max }}: \mathrm{kN}$ & $\tau_{\max }: \mathrm{MPa}$ & $\tau_{0.1}: \mathrm{MPa}$ & $W_{15}: \mathrm{mm}$ & Failure mode & $\begin{array}{c}\tau_{\max , a \mathrm{av}}: \\
\mathrm{MPa}\end{array}$ & $\begin{array}{c}\tau_{\text {max,ava }} / f_{\text {bd }} \\
(\mathrm{MC} 2010)\end{array}$ \\
\hline
\end{tabular}

\begin{tabular}{|c|c|c|c|c|c|c|c|c|}
\hline \multicolumn{9}{|c|}{ Series $4, f_{\mathrm{Rm}}=0.081, f_{\mathrm{cm}}=47.8 \mathrm{MPa}, d=12 \mathrm{~mm}$} \\
\hline 25 & 0.076 & $55 \cdot 8$ & $24 \cdot 68$ & $13 \cdot 46$ & - & SPL & \multirow[t]{8}{*}{28.9} & \multirow[t]{8}{*}{$8 \cdot 39$} \\
\hline 26 & 0.084 & $65 \cdot 1$ & $28 \cdot 78$ & $18 \cdot 20$ & 0.014 & SPL & & \\
\hline 27 & 0.083 & $68 \cdot 1$ & $30 \cdot 09$ & $18 \cdot 62$ & 0.021 & SPL & & \\
\hline 28 & 0.082 & $66 \cdot 6$ & $29 \cdot 45$ & $19 \cdot 24$ & 0.018 & SPL & & \\
\hline 29 & 0.077 & $60 \cdot 3$ & $26 \cdot 65$ & $14 \cdot 45$ & 0.019 & SPL & & \\
\hline 30 & 0.075 & $63 \cdot 1$ & $27 \cdot 92$ & $14 \cdot 68$ & 0.008 & $\mathrm{PO}$ & & \\
\hline 31 & 0.096 & $76 \cdot 8$ & $33 \cdot 96$ & $21 \cdot 44$ & 0.004 & SPL & & \\
\hline 32 & 0.079 & $67 \cdot 3$ & $29 \cdot 74$ & $15 \cdot 06$ & 0.017 & SPL & & \\
\hline \multicolumn{9}{|c|}{ Series $5, f_{\mathrm{Rm}}=0.095, f_{\mathrm{cm}}=47.8 \mathrm{MPa}, d=16 \mathrm{~mm}$} \\
\hline 33 & 0.086 & $103 \cdot 9$ & $25 \cdot 83$ & $9 \cdot 89$ & 0.013 & SPL & \multirow[t]{8}{*}{$25 \cdot 5$} & \multirow[t]{8}{*}{$7 \cdot 41$} \\
\hline 34 & 0.095 & $91 \cdot 4$ & $22 \cdot 73$ & $14 \cdot 35$ & 0.034 & SPL & & \\
\hline 35 & 0.097 & $100 \cdot 8$ & $25 \cdot 06$ & $14 \cdot 70$ & 0.013 & SPL & & \\
\hline 36 & 0.096 & $104 \cdot 8$ & $26 \cdot 05$ & $12 \cdot 94$ & 0.009 & SPL & & \\
\hline 37 & 0.105 & $121 \cdot 4$ & $30 \cdot 18$ & $15 \cdot 64$ & 0.011 & SPL & & \\
\hline 38 & 0.094 & $114 \cdot 7$ & $28 \cdot 53$ & $13 \cdot 03$ & 0.009 & SPL & & \\
\hline 39 & 0.097 & 85.4 & $21 \cdot 24$ & 14.65 & 0.022 & SPL & & \\
\hline 40 & 0.093 & $98 \cdot 5$ & $24 \cdot 49$ & $13 \cdot 06$ & 0.010 & SPL & & \\
\hline \multicolumn{9}{|c|}{ Series $6, f_{\mathrm{Rm}}=0.092, f_{\mathrm{cm}}=47.8 \mathrm{MPa}, d=20 \mathrm{~mm}$} \\
\hline 41 & 0.089 & $131 \cdot 1$ & $20 \cdot 87$ & $12 \cdot 60$ & 0.011 & SPL & \multirow[t]{8}{*}{$25 \cdot 5$} & \multirow[t]{8}{*}{$7 \cdot 41$} \\
\hline 42 & $0 \cdot 100$ & $157 \cdot 9$ & $25 \cdot 13$ & $16 \cdot 98$ & 0.001 & SPL & & \\
\hline 43 & 0.086 & $167 \cdot 6$ & $26 \cdot 67$ & $10 \cdot 90$ & 0.025 & SPL & & \\
\hline 44 & 0.094 & $175 \cdot 0$ & $27 \cdot 85$ & $16 \cdot 42$ & 0.025 & SPL & & \\
\hline 45 & 0.090 & $144 \cdot 3$ & $22 \cdot 97$ & $11 \cdot 90$ & 0.017 & SPL & & \\
\hline 46 & 0.098 & $174 \cdot 7$ & $27 \cdot 80$ & $11 \cdot 85$ & 0.015 & SPL & & \\
\hline 47 & 0.091 & - & - & - & - & - & & \\
\hline 48 & 0.091 & $171 \cdot 7$ & $27 \cdot 32$ & $12 \cdot 86$ & 0.012 & SPL & & \\
\hline \multicolumn{9}{|c|}{ Series 7, $f_{\mathrm{Rm}}=0.047, f_{\mathrm{cm}}=63.4 \mathrm{MPa}, d=12 \mathrm{~mm}$} \\
\hline 49 & 0.043 & $67 \cdot 85$ & $30 \cdot 00$ & $18 \cdot 02$ & 0.003 & SPL & \multirow[t]{8}{*}{$33 \cdot 6$} & \multirow[t]{8}{*}{$8 \cdot 32$} \\
\hline 50 & 0.058 & $76 \cdot 68$ & $33 \cdot 90$ & $18 \cdot 71$ & 0.005 & SPL & & \\
\hline 51 & 0.042 & $65 \cdot 14$ & $28 \cdot 80$ & $16 \cdot 71$ & 0.012 & SPL & & \\
\hline 52 & 0.057 & $75 \cdot 82$ & $33 \cdot 52$ & $19 \cdot 07$ & 0.020 & SPL & & \\
\hline 53 & 0.040 & $79 \cdot 18$ & $35 \cdot 01$ & $18 \cdot 16$ & 0.009 & SPL & & \\
\hline 54 & 0.052 & $89 \cdot 44$ & $39 \cdot 54$ & $16 \cdot 29$ & 0.011 & SPL & & \\
\hline 55 & 0.043 & $82 \cdot 35$ & $36 \cdot 41$ & $21 \cdot 48$ & 0.004 & SPL & & \\
\hline 56 & 0.040 & 71.66 & $31 \cdot 68$ & $15 \cdot 18$ & 0.016 & SPL & & \\
\hline \multicolumn{9}{|c|}{ Series $8, f_{\mathrm{Rm}}=0.061, f_{\mathrm{cm}}=63.4 \mathrm{MPa}, d=16 \mathrm{~mm}$} \\
\hline 57 & 0.058 & $122 \cdot 9$ & $30 \cdot 55$ & $17 \cdot 25$ & 0.002 & SPL & \multirow[t]{8}{*}{$28 \cdot 3$} & \multirow[t]{8}{*}{$7 \cdot 03$} \\
\hline 58 & 0.065 & $147 \cdot 5$ & $36 \cdot 68$ & $19 \cdot 65$ & 0.009 & SPL & & \\
\hline 59 & 0.056 & $124 \cdot 6$ & $30 \cdot 99$ & $20 \cdot 18$ & 0.009 & SPL & & \\
\hline 60 & 0.061 & $118 \cdot 0$ & $29 \cdot 35$ & $20 \cdot 92$ & 0.034 & SPL & & \\
\hline 61 & 0.059 & $80 \cdot 26$ & $19 \cdot 96$ & $19 \cdot 91$ & 0.035 & SPL & & \\
\hline 62 & 0.063 & $100 \cdot 3$ & 24.95 & $19 \cdot 32$ & 0.035 & $\mathrm{PO}$ & & \\
\hline 63 & 0.058 & $107 \cdot 1$ & $26 \cdot 64$ & $19 \cdot 78$ & 0.016 & $\mathrm{PO}$ & & \\
\hline 64 & 0.066 & $110 \cdot 4$ & $27 \cdot 47$ & $20 \cdot 64$ & - & $\mathrm{PO}$ & & \\
\hline
\end{tabular}

Table 6. Summary of test results of machined bars (continued on next page) 


\begin{tabular}{|c|c|c|c|c|c|c|c|c|}
\hline Specimen & $f_{\mathrm{R}}$ & $P_{\max }: \mathrm{kN}$ & $\tau_{\max }: \mathrm{MPa}$ & $\tau_{0 \cdot 1}: \mathrm{MPa}$ & $W_{15}: \mathrm{mm}$ & Failure mode & $\begin{array}{c}\tau_{\max , a v}: \\
\mathrm{MPa}\end{array}$ & $\begin{array}{c}\tau_{\max , \mathrm{av}} / f_{\mathrm{bd}} \\
(\mathrm{MC} 2010)\end{array}$ \\
\hline \multicolumn{9}{|c|}{ Series $9, f_{\mathrm{Rm}}=0.066, f_{\mathrm{cm}}=63.4 \mathrm{MPa}, d=20 \mathrm{~mm}$} \\
\hline 65 & 0.063 & $168 \cdot 2$ & $26 \cdot 78$ & $15 \cdot 99$ & 0.012 & $\mathrm{SPL}$ & \multirow[t]{8}{*}{$25 \cdot 5$} & \multirow[t]{8}{*}{$6 \cdot 31$} \\
\hline 66 & 0.073 & $155 \cdot 8$ & $24 \cdot 81$ & $21 \cdot 68$ & 0.015 & SPL & & \\
\hline 67 & 0.068 & $198 \cdot 9$ & $31 \cdot 66$ & $17 \cdot 96$ & 0.019 & SPL & & \\
\hline 68 & 0.049 & $142 \cdot 2$ & $22 \cdot 64$ & $14 \cdot 70$ & 0.027 & SPL & & \\
\hline 69 & 0.066 & $185 \cdot 2$ & $29 \cdot 47$ & $17 \cdot 34$ & 0.017 & $\mathrm{PO}$ & & \\
\hline 70 & 0.069 & $157 \cdot 4$ & $25 \cdot 06$ & $14 \cdot 80$ & 0.019 & PO & & \\
\hline 71 & 0.063 & $127 \cdot 3$ & $20 \cdot 26$ & $15 \cdot 21$ & 0.022 & $\mathrm{PO}$ & & \\
\hline 72 & 0.069 & $145 \cdot 4$ & $23 \cdot 14$ & $16 \cdot 36$ & 0.026 & PO & & \\
\hline \multicolumn{9}{|c|}{ Series $10, f_{\mathrm{Rm}}=0.081, f_{\mathrm{cm}}=74.3 \mathrm{MPa}, d=12 \mathrm{~mm}$} \\
\hline 73 & 0.076 & $86 \cdot 1$ & 38.06 & $30 \cdot 06$ & 0.008 & SPL & \multirow[t]{8}{*}{$36 \cdot 3$} & \multirow[t]{8}{*}{$8 \cdot 22$} \\
\hline 74 & 0.084 & $85 \cdot 8$ & 37.92 & $20 \cdot 06$ & 0.006 & SPL & & \\
\hline 75 & 0.083 & $84 \cdot 1$ & $37 \cdot 19$ & $23 \cdot 00$ & 0.015 & $\mathrm{SPL}$ & & \\
\hline 76 & 0.082 & 93.9 & $41 \cdot 51$ & $30 \cdot 93$ & 0.011 & SPL & & \\
\hline 77 & 0.077 & $60 \cdot 1$ & $26 \cdot 57$ & - & - & SPL & & \\
\hline 78 & 0.075 & $97 \cdot 5$ & $43 \cdot 11$ & $29 \cdot 46$ & 0.008 & $\mathrm{SPL}$ & & \\
\hline 79 & 0.096 & $64 \cdot 3$ & $28 \cdot 42$ & $23 \cdot 11$ & 0.006 & $\mathrm{SPL}$ & & \\
\hline 80 & 0.079 & 85.9 & 37.96 & $20 \cdot 18$ & 0.015 & $\mathrm{SPL}$ & & \\
\hline \multicolumn{9}{|c|}{ Series $11, f_{\mathrm{Rm}}=0.095, f_{\mathrm{cm}}=74.3 \mathrm{MPa}, d=16 \mathrm{~mm}$} \\
\hline 81 & 0.086 & $147 \cdot 5$ & $36 \cdot 67$ & $31 \cdot 70$ & 0.011 & $\mathrm{SPL}$ & \multirow[t]{8}{*}{$35 \cdot 9$} & \multirow[t]{8}{*}{$8 \cdot 12$} \\
\hline 82 & 0.095 & $163 \cdot 8$ & $40 \cdot 71$ & $25 \cdot 25$ & 0.007 & SPL & & \\
\hline 83 & 0.097 & $117 \cdot 4$ & $29 \cdot 20$ & $28 \cdot 87$ & 0.010 & SPL & & \\
\hline 84 & 0.096 & $156 \cdot 7$ & 38.96 & $31 \cdot 73$ & 0.019 & SPL & & \\
\hline $85^{a}$ & $0 \cdot 105$ & $93 \cdot 8$ & $23 \cdot 33$ & - & 0.012 & SPL & & \\
\hline 86 & 0.094 & $116 \cdot 7$ & $29 \cdot 02$ & $29 \cdot 02$ & 0.014 & SPL & & \\
\hline 87 & 0.097 & $143 \cdot 9$ & $35 \cdot 80$ & $34 \cdot 29$ & 0.016 & SPL & & \\
\hline 88 & 0.093 & $164 \cdot 9$ & $41 \cdot 00$ & $32 \cdot 67$ & 0.013 & SPL & & \\
\hline \multicolumn{9}{|c|}{ Series $12, f_{\mathrm{Rm}}=0.092, f_{\mathrm{cm}}=74.3 \mathrm{MPa}, d=20 \mathrm{~mm}$} \\
\hline 89 & 0.089 & $204 \cdot 5$ & $32 \cdot 55$ & $23 \cdot 69$ & 0.016 & SPL & \multirow[t]{8}{*}{$33 \cdot 8$} & \multirow[t]{8}{*}{$7 \cdot 64$} \\
\hline 90 & $0 \cdot 100$ & $213 \cdot 7$ & $34 \cdot 01$ & $24 \cdot 59$ & 0.027 & SPL & & \\
\hline 91 & 0.086 & $238 \cdot 4$ & 37.94 & $20 \cdot 17$ & 0.016 & SPL & & \\
\hline 92 & 0.094 & $243 \cdot 5$ & $38 \cdot 76$ & $30 \cdot 24$ & 0.015 & SPL & & \\
\hline 93 & 0.090 & $199 \cdot 2$ & $31 \cdot 70$ & $30 \cdot 28$ & 0.016 & SPL & & \\
\hline 94 & 0.098 & $222 \cdot 4$ & $35 \cdot 40$ & 35.09 & 0.008 & SPL & & \\
\hline $95^{a}$ & 0.091 & $131 \cdot 0$ & $20 \cdot 84$ & - & 0.019 & SPL & & \\
\hline 96 & 0.091 & $165 \cdot 4$ & $26 \cdot 33$ & $25 \cdot 9$ & 0.022 & SPL & & \\
\hline
\end{tabular}

a Disregarded result.

SPL, splitting failure; PO, pull-out failure.

Table 6. (continued)

Effect of bond index and bar diameter on bond strength Test results concerning the bond strength $\tau_{\max }$ are summarised in Figures 7-9; the results of each specimen with a machined rebar are plotted individually against the bond index $f_{\mathrm{R}}$ for both NSC (Figure 7(a)) and HSC specimens (Figure 7(b)). The mean bond strength of each series with hot-rolled commercial bars is also plotted in Figures 7-9. It is worth noting that the bond strength increases with the bond index and decreases with bar diameter. The increase in bond strength with bond index is more evident for the larger diameter rebars due to a stronger interaction between the ribs and the surrounding concrete; for smaller rebars, this interaction is limited by the porous concrete layer present in front of the ribs (Giuriani, 1982). The hot-rolled commercial bars showed a behaviour similar to the machined rebars. The larger bars, tested only in NSC specimens, exhibited a reduced bond strength with average values of $15 \cdot 8-17.9 \mathrm{MPa}$ for the $40 \mathrm{~mm}$ diameter bars and $16 \cdot 3-18 \cdot 3 \mathrm{MPa}$ for the $50 \mathrm{~mm}$ diameter bars. 


\begin{tabular}{|c|c|c|c|c|c|c|c|c|}
\hline Specimen & $f_{\mathrm{R}}$ & $P_{\max }: \mathrm{kN}$ & $\tau_{\text {max }}: \mathrm{MPa}$ & $\tau_{0 \cdot 1}: \mathrm{MPa}$ & $W_{15}: \mathrm{mm}$ & Failure mode & $\begin{array}{c}\tau_{\max , a v}: \\
M P a\end{array}$ & $\begin{array}{c}\tau_{\text {max }, \text { av }} / f_{\mathrm{bd}} \\
(\mathrm{MC} 2010)\end{array}$ \\
\hline
\end{tabular}

Series $13, f_{\mathrm{Rm}}=0.095, f_{\mathrm{cm}}=41.5 \mathrm{MPa}, d=12 \mathrm{~mm}$

$\begin{array}{llll}97 & 0.095 & 46.5 & 20.54\end{array}$

$98 \quad 0.095 \quad 56.4 \quad 24.93$

$\begin{array}{llll}99 & 0.095 & 59.4 & 26.26\end{array}$

$\begin{array}{llll}101 & 0.095 & 62.4 & 27.58\end{array}$

$\begin{array}{llll}102^{\mathrm{a}} & 0.095 & 34.4 & 15.20\end{array}$

$103 \quad 0.095 \quad 54.5 \quad 24.10$

$104 \quad 0.095 \quad 44.3 \quad 19.59$

Series $14, f_{\mathrm{Rm}}=0.089, f_{\mathrm{cm}}=41.5 \mathrm{MPa}, d=20 \mathrm{~mm}$

$\begin{array}{llll}105 & 0.089 & 128.3 & 20.42\end{array}$

$\begin{array}{llll}107 & 0.089 & 137.8 & 21.94\end{array}$

$\begin{array}{llll}108 & 0.089 & 122.1 & 19.43\end{array}$

109

110

111

0.089

123.9

$19 \cdot 71$

0.089

$128 \cdot 2$

$20 \cdot 40$

$19 \cdot 59$

Series $15, f_{\mathrm{Rm}}=0.105, f_{\mathrm{cm}}=64.8 \mathrm{MPa}, d=12 \mathrm{~mm}$

$\begin{array}{llll}112 & 0.105 & 76.9 & 33.99 \\ 113 & 0.105 & 57.5 & 25.40\end{array}$

113

114

115

116

117

118

119

Series $16, f_{\mathrm{R}}=0.079, f_{\mathrm{m}}$

$0 \cdot 105$

80.0

25.40
35.35

0.105

$79 \cdot 3$

$35 \cdot 35$

$0 \cdot 105$

75.8

35.07

0.105

0.105

$$
-
$$

\section{$77 \cdot 3$}

33.53

67.6

34. 15

29.90

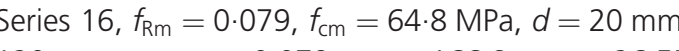

$\begin{array}{llll}120 & 0.079 & 166.8 & 26.55 \\ 121 & 0.079 & 170.1 & 27.07\end{array}$

122

123

124

125

126

127

0.079

0.079

$166 \cdot 4$

$26 \cdot 48$

0.079

$163 \cdot 1$

$25 \cdot 95$

$26 \cdot 98$

0.079

$169 \cdot 5$

$27 \cdot 32$

27.04

0.079

169.9

$169 \cdot 5$

$26 \cdot 98$

$128 \quad 0.054 \quad 391.8 \quad 15.59$

$\begin{array}{llll}129 & 0.054 & 299.8 & 11.93\end{array}$

130

0.054

248.8

9.90

0.054

$547 \cdot 4$

$21 \cdot 78$

$0.054 \quad 502.1 \quad 19.98$

132

Series

133

134

135

136

137

138

139

0.07

$072, f_{\mathrm{cm}}=37.6 \mathrm{MPa}, d=40 \mathrm{~mm}$

$\begin{array}{lll}0.072 & 439.6 & 17.49\end{array}$

0.072

$431 \cdot 0$

$17 \cdot 15$

0.072

0.072

403.9

$16 \cdot 07$

19.91

0.072

$500 \cdot 4$

18.77

$431 \cdot 3$

$17 \cdot 16$ $\begin{array}{llll}100 & 0.095 & 41.7 & 18.44\end{array}$

$\begin{array}{llll}106 & 0.089 & 150 \cdot 7 & 23.98\end{array}$
16.57

15.48

14.11

$16 \cdot 17$

$18 \cdot 17$

9.85

17.08

10.32

$10 \cdot 91$

10.85

10.83

8.56

$12 \cdot 40$

13.20

9.44

33.27

27.58

31.09

31.90

$-$

29.87

24.83

25.06

22.60

21.41

26.37

22.73

24. 51

23.68

4.68

3.63

4.66

4.01

4.39

$2 \cdot 50$

3.90

3.85

4. 56

$7 \cdot 27$

3.67

4.53
0.007

0.007

0.011

0.024

0.009

$\overline{0.013}$

0.026

$\begin{array}{cc}- & P O \\ 0.027 & \text { SPL } \\ 0.038 & \text { SPL } \\ 0.038 & \text { PO } \\ 0.030 & \text { SPL } \\ 0.025 & \text { SPL } \\ 0.021 & \text { SPL }\end{array}$

0.018

0.007

0.017

0.003

0.010

-

0.005

0.020

0.031

0.028

0.037

0.028

0.026

0.036

0.037

SPL

SPL

SPL

SPL

PO

SPL

SPL

SPL

PO

SPL

PO

SPL

SPL

SPL

PO

Y

$Y$

SPL

SPL

SPL

SPL

SPL
Y

SPL

SPL

$Y$
$Y$

Y

Y

$\begin{array}{ll}- & \mathrm{SPL} \\ - & \mathrm{SPL} \\ - & \mathrm{SPL} \\ - & \mathrm{SPL} \\ - & \mathrm{SPL}\end{array}$

$26 \cdot 8$

$6 \cdot 64$

SPL

SPL

SPL

SPL

$\begin{array}{ll}- & \mathrm{SPL} \\ - & \mathrm{SPL} \\ - & \mathrm{PO} \\ - & \mathrm{PO} \\ - & \mathrm{SPL} \\ - & \mathrm{SPL} \\ - & \mathrm{SPL}\end{array}$

$23 \cdot 1$

$7 \cdot 16$

$20 \cdot 8$

$6 \cdot 45$

$32 \cdot 5$

8.04
Table 7. Summary of test results of commercial bars (continued on next page) 


\begin{tabular}{|c|c|c|c|c|c|c|c|c|}
\hline Specimen & $f_{\mathrm{R}}$ & $P_{\max }: \mathrm{kN}$ & $\tau_{\max }: \mathrm{MPa}$ & $\tau_{0 \cdot 1}: \mathrm{MPa}$ & $W_{15}: \mathrm{mm}$ & Failure mode & $\begin{array}{c}\tau_{\max , a v}: \\
\mathrm{MPa}\end{array}$ & $\begin{array}{c}\tau_{\max , a v} / f_{\mathrm{bd}} \\
(\mathrm{MC} 2010)\end{array}$ \\
\hline \multicolumn{9}{|c|}{ Series $19, f_{\mathrm{Rm}}=0.040, f_{\mathrm{cm}}=37.6 \mathrm{MPa}, d=50 \mathrm{~mm}$} \\
\hline 140 & 0.040 & $688 \cdot 8$ & $13 \cdot 93$ & $2 \cdot 00$ & - & SPL & \multirow[t]{6}{*}{$16 \cdot 3$} & \multirow[t]{6}{*}{$6 \cdot 74$} \\
\hline 141 & 0.040 & $663 \cdot 3$ & $14 \cdot 49$ & $5 \cdot 22$ & - & SPL & & \\
\hline 142 & 0.040 & $799 \cdot 5$ & $14 \cdot 77$ & $1 \cdot 23$ & - & SPL & & \\
\hline 143 & 0.040 & $777 \cdot 9$ & $18 \cdot 81$ & $4 \cdot 32$ & - & $\mathrm{SPL}$ & & \\
\hline 144 & 0.040 & $726 \cdot 1$ & $18 \cdot 85$ & $4 \cdot 32$ & - & SPL & & \\
\hline 145 & 0.040 & $547 \cdot 0$ & $17 \cdot 04$ & $3 \cdot 01$ & - & SPL & & \\
\hline \multicolumn{9}{|c|}{ Series $20, f_{\mathrm{Rm}}=0.063, f_{\mathrm{cm}}=37.6 \mathrm{MPa}, d=50 \mathrm{~mm}$} \\
\hline 146 & 0.063 & $612 \cdot 2$ & $16 \cdot 66$ & $3 \cdot 23$ & - & $\mathrm{SPL}$ & \multirow[t]{6}{*}{$18 \cdot 3$} & \multirow[t]{6}{*}{$7 \cdot 55$} \\
\hline 147 & 0.063 & $468 \cdot 5$ & $17 \cdot 54$ & $2 \cdot 32$ & - & $\mathrm{SPL}$ & & \\
\hline 148 & 0.063 & $388 \cdot 8$ & $16 \cdot 89$ & $3 \cdot 56$ & - & SPL & & \\
\hline 149 & 0.063 & $855 \cdot 3$ & $20 \cdot 36$ & $6 \cdot 36$ & - & $\mathrm{SPL}$ & & \\
\hline 150 & 0.063 & 784.6 & $19 \cdot 81$ & $5 \cdot 25$ & - & SPL & & \\
\hline 151 & 0.063 & $654 \cdot 2$ & 18.49 & 3.75 & - & $\mathrm{SPL}$ & & \\
\hline
\end{tabular}

${ }^{a}$ Disregarded result.

SPL, splitting failure; PO, pull-out failure; $Y$, bar yielding.

Table 7. (continued)

Since the concrete strength $f_{\mathrm{cm} \text {,cube }}$ varies between 35.5 and $74.3 \mathrm{MPa}$ and it is widely recognised that bond strength is proportional to the tensile strength of concrete, the test results were normalised to the power of $2 / 3$ of the compressive strength to allow for a better comparison between specimens made of different materials (Figure 8). By means of linear regressions of the experimental results, it can be seen that by increasing the relative rib area from 0.04 to $0 \cdot 105$, the bond strength of the $20 \mathrm{~mm}$ rebars increased by up to $40 \%$. Conversely, within the same range of bond index, specimens with a $12 \mathrm{~mm}$ rebar showed a fairly constant bond strength.

The bond behaviour is strongly affected by bar diameter, as noted in previous studies on the size effect of highly confined ribbed bars with a short embedded length (Bamonte and Gambarova, 2007; Bazant and Sener, 1988); in fact, the bigger the bar diameter the lower the bond strength. Figure 9 shows the mean value of the normalised bond strength $\left(\tau_{\text {max,av }} / f_{\text {cm,cube }}^{2 / 3}\right)$ of each series (both machined and commercial) in NSC plotted against bar diameter. It can be observed that the bond strength decreases by about $26 \%$ when the bar diameter increases from 12 to $50 \mathrm{~mm}$ and about by $13 \%$ for diameters from 20 to $50 \mathrm{~mm}$ (the cover/diameter ratio and the bond length/diameter ratio of all tested specimens being constant). One may also note the limited reduction in bond strength of the larger bars (40 and $50 \mathrm{~mm}$ ) with a bond index lower than the minimum value required by Eurocode 2. These results are consistent with those of the pull-out tests presented by Ichinose et al. (2004), which showed a reduction in average bond strength from $12 \%$ to $54 \%$ with an increase in bar diameter from 17 to $50 \mathrm{~mm}$; a larger difference was found in tests on bars with a higher relative rib area $\left(f_{\mathrm{R}}>0 \cdot 3\right)$ and cover thickness equal to only $1 \cdot 22$ times the bar diameter due to splitting phenomena.

\section{Comparison with the formulation proposed in MC2010}

Since the tests were carried out with a short anchorage length (five times the bar diameter), the experimental bond-slip curve can be a useful tool to investigate the effectiveness of the local bond-slip law proposed by MC2010 (fib, 2013). For monotonic loading and for splitting failure in good bond conditions, the bond stress is calculated as a function of the slip $\delta$ according to

4. $\tau=\tau_{\mathrm{bmax}}\left(\delta / \delta_{1}\right)^{0 \cdot 4} \leqslant \tau_{\mathrm{u}, \text { split }}$

where $\delta_{1}=1 \mathrm{~mm}, \tau_{\text {bmax }}$ is the maximum bond stress for a pullout failure, given by

5. $\tau_{\text {bmax }}=2 \cdot 5 f_{\mathrm{cm}}^{0 \cdot 5}$

$\tau_{\mathrm{u}, \text { split }}$ is the bond strength for splitting failure, given by the following equation when transverse reinforcement is not present

6. $\tau_{\mathrm{u}, \mathrm{split}}=6 \cdot 5\left(\frac{f_{\mathrm{cm}}}{25}\right)^{0.25}\left(\frac{25}{d}\right)^{0 \cdot 20}\left[\left(\frac{c_{\min }}{d}\right)^{0.33}\left(\frac{c_{\max }}{c_{\text {min }}}\right)^{0 \cdot 10}\right]$

in which $c_{\max }$ and $c_{\min }$ are the maximum and minimum concrete cover (both equal to $4 \cdot 5 d$ in the present tests; Figure 2). 
Influence of the relative rib area on bond

behaviour

Metelli and Plizzari

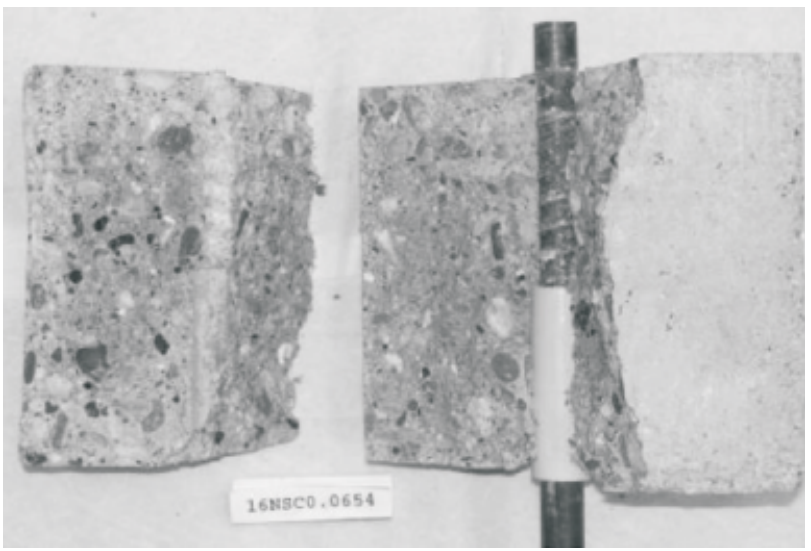

(a)

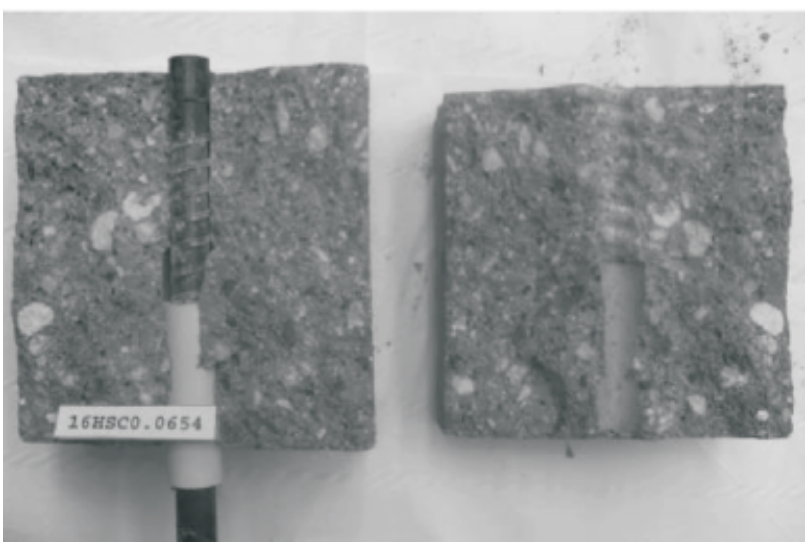

(b)

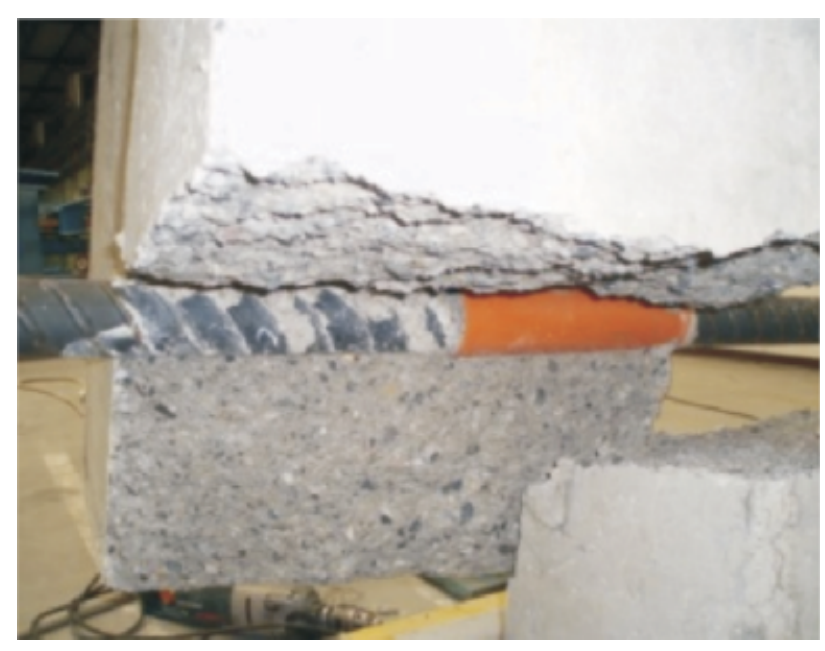

(c)

Figure 4. Typical splitting failure for normal-size bars in (a) NSC and (b) HSC. (c) Typical splitting failure for large-diameter bars

Figure 5 shows significant differences between the experimental bond-slip curves and the local bond-slip law proposed by MC2010, in terms of maximum bond strength, stiffness and slip at failure; these differences increase with the concrete strength and for small bar diameter. The MC2010 formulation markedly underestimates bond strength when smaller rebars $(12 \mathrm{~mm})$ with a higher bond index are used in HSC. Nevertheless, as shown in Tables 6 and 7, the mean bond strength of each series was always significantly greater than the design bond strength $f_{\text {bd }}$ proposed by MC2010, as shown by

7. $f_{\mathrm{bd}}=\alpha_{2} f_{\mathrm{bd}, 0}<2.5 f_{\mathrm{bd}, 0}<1.5 \frac{f_{\mathrm{ck}}^{0.5}}{\gamma_{\mathrm{cb}}}$

where $\alpha_{2}$ is a factor that takes into account the confining effects of concrete and $f_{\mathrm{bd}, 0}$ is the basic bond strength

8. $\alpha_{2}=(c / d)^{0 \cdot 5}=2 \cdot 12$

9. $f_{\mathrm{bd}, 0}=\frac{\eta_{1} \eta_{2} \eta_{3} \eta_{4}\left(f_{\mathrm{ck}} / 25\right)^{0 \cdot 5}}{\gamma_{\mathrm{cb}}}$

in which the concrete cover in the pull-out specimen $c=4 \cdot 5 d$, $\eta_{1}=1.75$ for ribbed bars, $\eta_{2}=1.00$ since good bond conditions are assumed, $\eta_{3}=(25 / d)^{0.3}, \eta_{4}=1.00-0.68$ depending on the steel grade $f_{\mathrm{yk}}$ ranging from 500 to $800 \mathrm{MPa}$ and $\gamma_{\mathrm{cb}}=1.5$ is the partial safety coefficient for bond.

As far as the machined bars are concerned, the experimental/ design strength ratio ranges between 6.31 for the $20 \mathrm{~mm}$ diameter bar $\left(f_{\mathrm{Rm}}=0.065\right)$ in NSC and 8.39 for the $12 \mathrm{~mm}$ diameter bar $\left(f_{\mathrm{Rm}}=0.081\right)$ in NSC. The hot-rolled bars showed similar values, even for large bars with a bond index below the limit prescribed by Eurocode $2\left(f_{\mathrm{R}}>0.056\right.$ if $\left.d>12 \mathrm{~mm}\right)$; in fact, the experimental/design strength ratio is 6.11 or 6.74 respectively for series 17 with $40 \mathrm{~mm}$ diameter bars $\left(f_{\mathrm{Rm}}=0.054\right)$ or series 19 with $50 \mathrm{~mm}$ diameter bars $\left(f_{\mathrm{Rm}}=0 \cdot 040\right)$.

In Figure 9, the test results are also compared with the bond strength calculated according to MC2010 and with the size-effect analytical formulation proposed by Bamonte and Gambarova (2007). It is worth noting that MC2010 provides safety values of the bond strength for design purposes, as previously discussed, whereas the Bamonte and Gambarova (2007) formulation accurately fits all the test results since it was specifically calibrated for short embedded lengths with confined conditions. Furthermore, the semi-empirical equation proposed by MC2010 is based on a database that does not include tests with anchorage lengths shorter than ten diameters, corresponding to the minimum value required by Eurocode 2 (fib, 2014).

\section{Effects on bond stiffness}

Figures 10 and 11, concerning the bond stress $\tau_{0 \cdot 1}$ measured at the unloaded-end slip value $\delta_{\mathrm{u}}=0 \cdot 1 \mathrm{~mm}$, provide useful information on the bond stiffness and, as a consequence, on the behaviour 
Influence of the relative rib area on bond

behaviour

Metelli and Plizzari
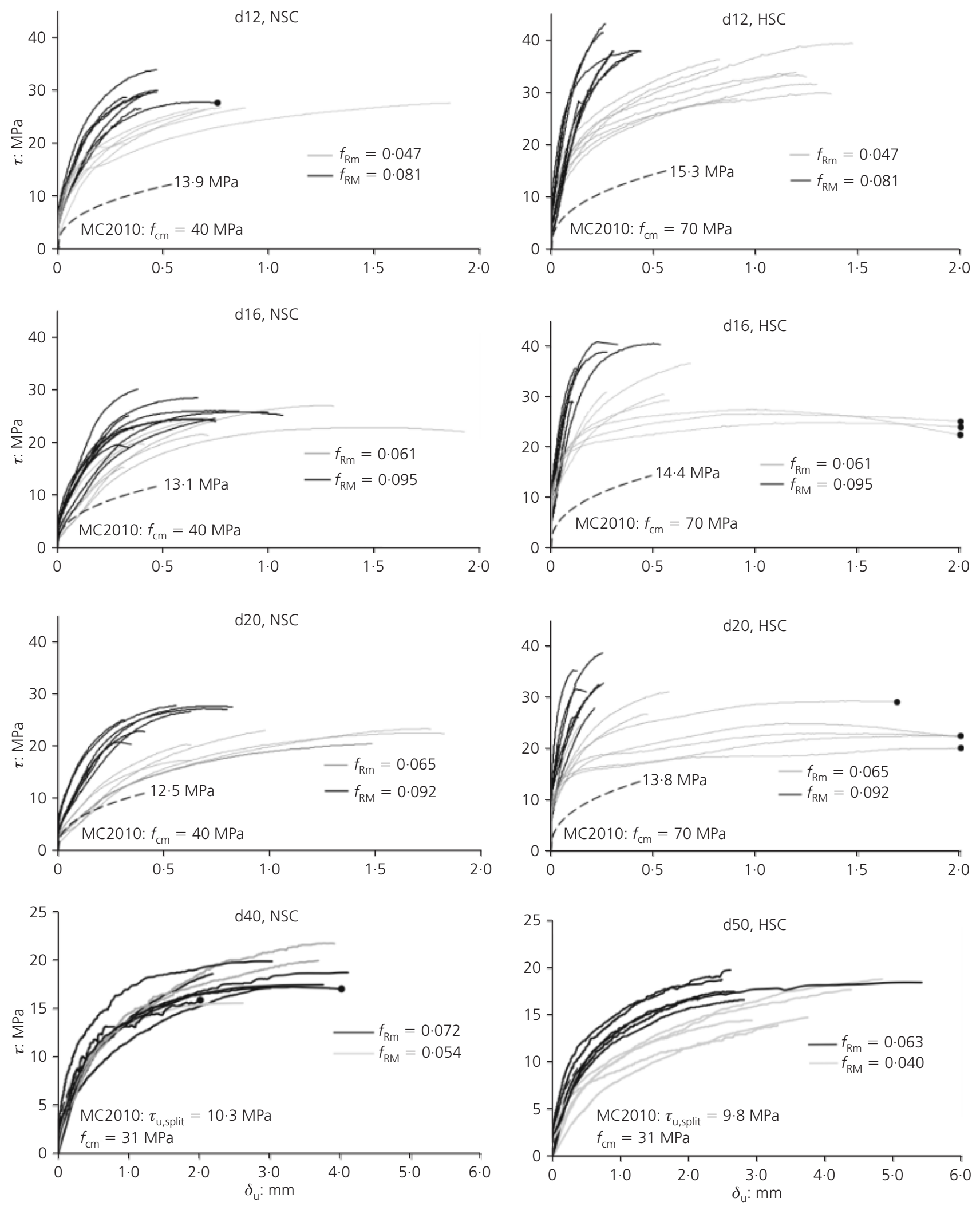

Figure 5. Bond stress $(\tau)$ plotted against unloaded-end slip $\left(\delta_{\mathrm{u}}\right)$ : comparison between test results of series with machined bars having different bond indexes but the same concrete strength $(\bullet$, pull-out failure). Notation d12, d16, etc., refers to bar diameter, in mm 

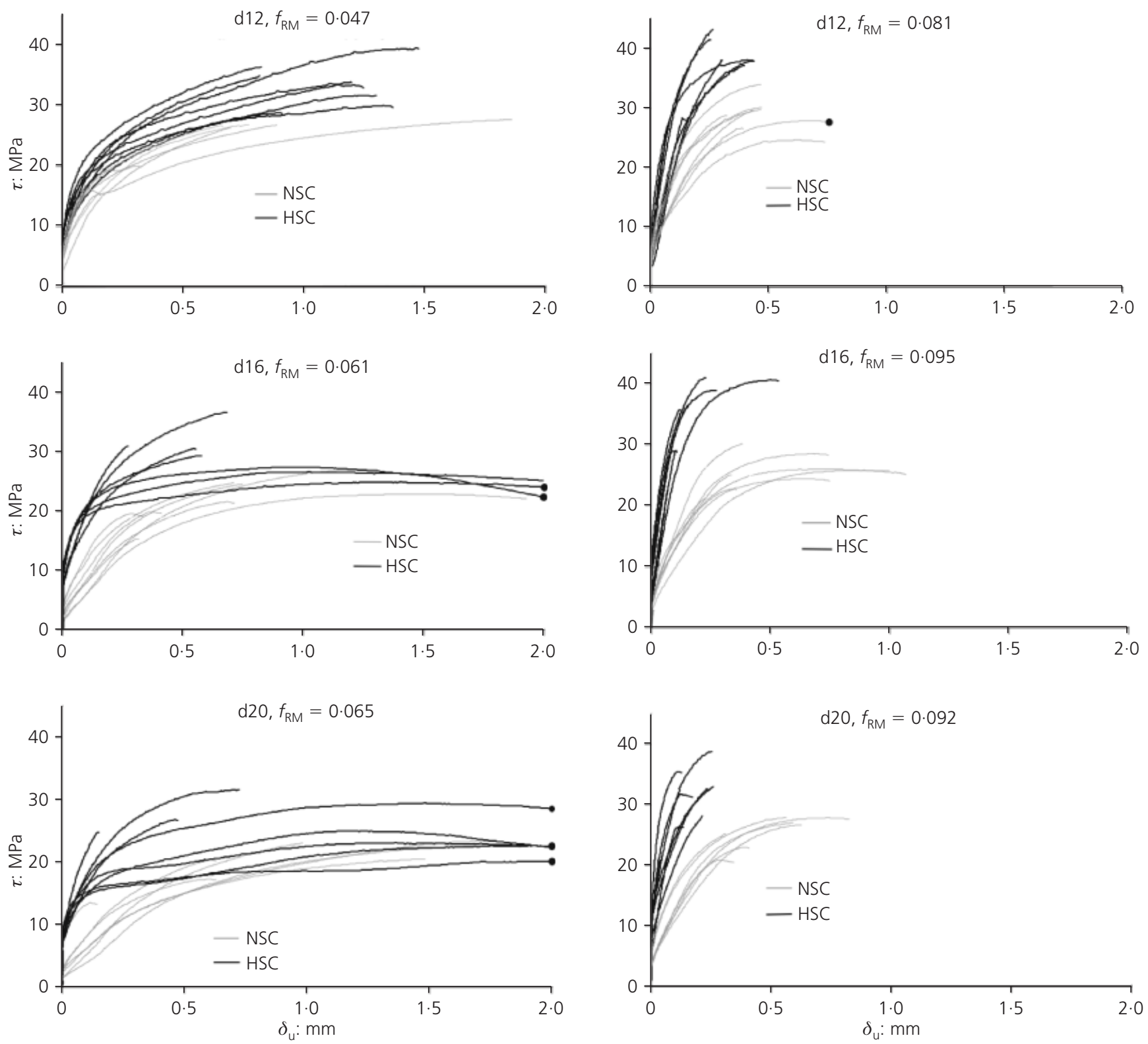

Figure 6. Bond stress $(\tau)$ plotted against unloaded-end slip $\left(\delta_{u}\right)$ : comparison between test results of series with machined bars having the same bond index but different concrete strength $(\bullet$, pull-out failure). Notation d12, d16, etc., refers to bar diameter, in mm

of the concrete member at service condition. Experimental results are plotted individually versus relative rib area $\left(f_{\mathrm{R}}\right)$, for both NSC and HSC specimens (Figure 10): the increase of the (secant) bond stiffness with both the relative rib area $f_{\mathrm{R}}$ (because of a more efficient interlocking between bar lugs and concrete) and the concrete strength (due to the lower porosity of HSC) can be noticed. For a given bond index, the bond stress $\tau_{0 \cdot 1}$ is about twice as great in HSC specimens than in NSC specimens, and this difference tends to increase with the bond index. This is also evident in Figure 6 where the bond stress-slip curves are compared between series with a different concrete strength but the same bond index and bar diameter. Furthermore, within the tested range of bond index (0.04-0.072), the 40 and $50 \mathrm{~mm}$ diameter rebars showed a fairly low bond stiffness.

The influence of bar diameter on bond stiffness is better evidenced in Figure 11 where $\tau_{0 \cdot 1}$ is plotted for each bar diameter $(12-50 \mathrm{~mm})$ in NSC; the experimental results show a clear size effect on bond stiffness, which is smaller in larger bars.

\section{Effect on wedging action}

In order to gain information on the bursting forces generated by the bar ribs, which tend to split the concrete cover around the bar, the transverse deformation of the cube specimens measured 
Influence of the relative rib area on bond

behaviour

Metelli and Plizzari

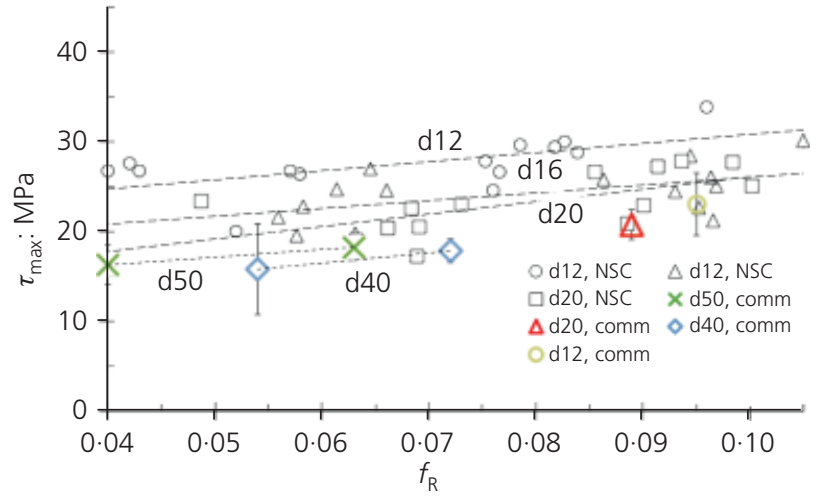

(a)

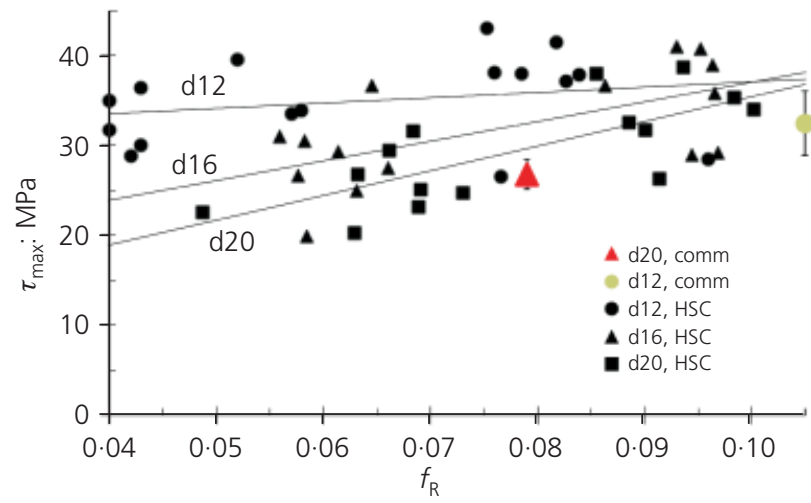

(b)

Figure 7. Bond strength $\left(\tau_{\max }\right)$ plotted against relative rib area $\left(f_{R}\right)$ for specimens in (a) NSC and (b) HSC. Notation d12, d16, etc. refers to bar diameter, in $\mathrm{mm}$; comm represents commercial hotrolled bar

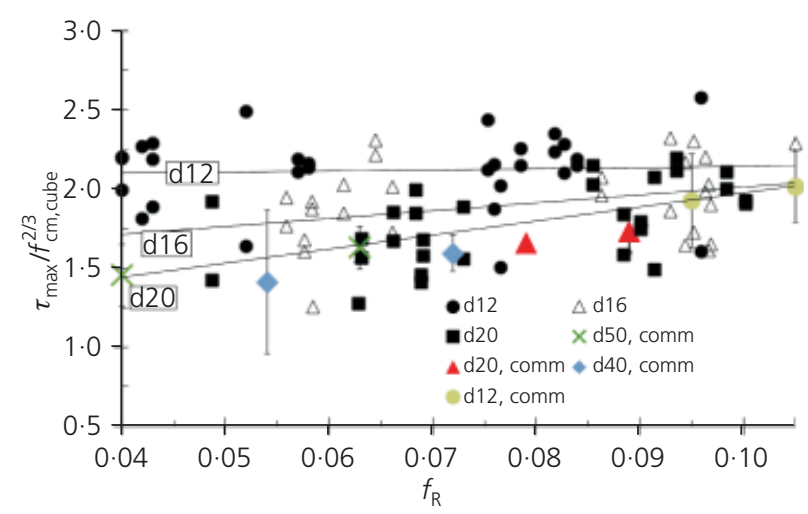

Figure 8. Normalised bond strength $\left(\tau_{\max }^{\prime}\right)$ plotted against relative rib area $\left(f_{R}\right)$. Notation d12, d16, etc. refers to bar diameter, in $\mathrm{mm}$; comm represents commercial hot-rolled bar

at an average bond stress of $15 \mathrm{MPa}\left(w_{15}\right)$ is plotted against relative rib area $f_{R}$ in Figure 12 for each specimen with machined rebars. The transverse deformation is calculated as the sum of the measurements given by the four LVDTs placed on each side of the specimen (Figure 2). Even though there is a wide scatter of

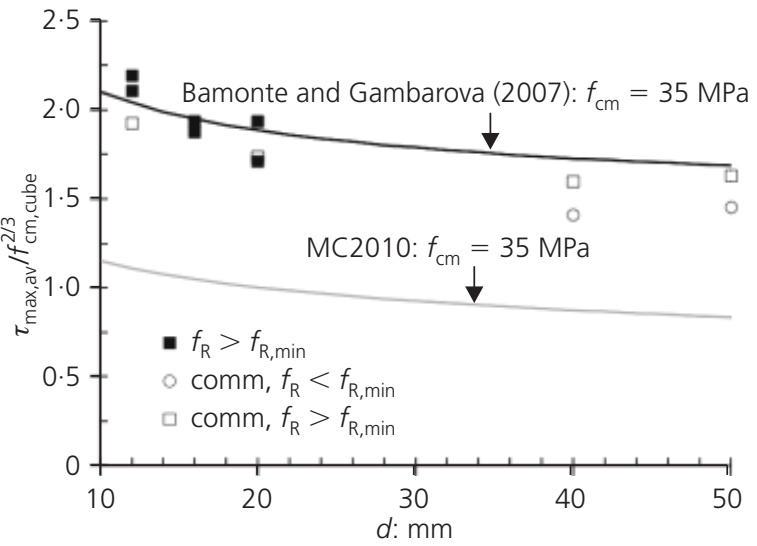

Figure 9. Normalised mean bond strength $\left(\tau_{\text {max,av }}^{\prime}\right)$ plotted against bar diameter $(d)$ and comparison with local bond-slip laws

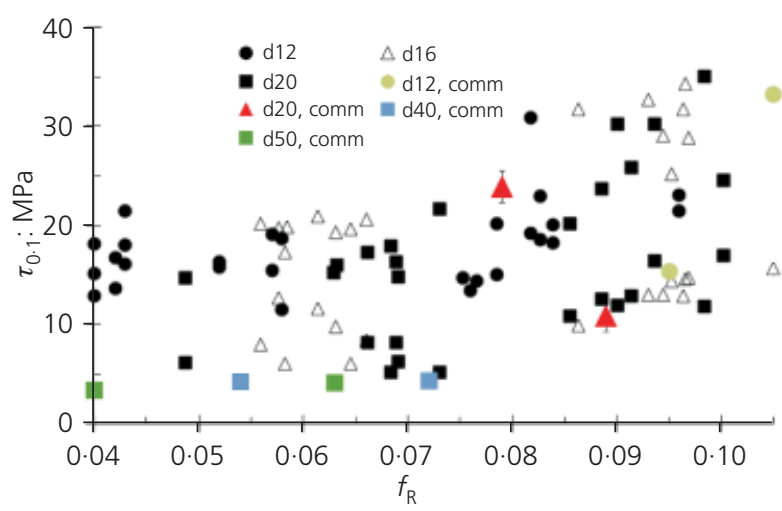

Figure 10. Influence of relative rib area on the bond stiffness: bond stress $\tau_{0.1}$ measured at unloaded-end slip $\left(\delta_{u}\right)$ of $0.1 \mathrm{~mm}$ plotted against relative rib area $\left(f_{\mathrm{R}}\right)$. Notation $\mathrm{d} 12$, d16, etc. refers to bar diameter, in $\mathrm{mm}$; comm represents commercial hot-rolled bar

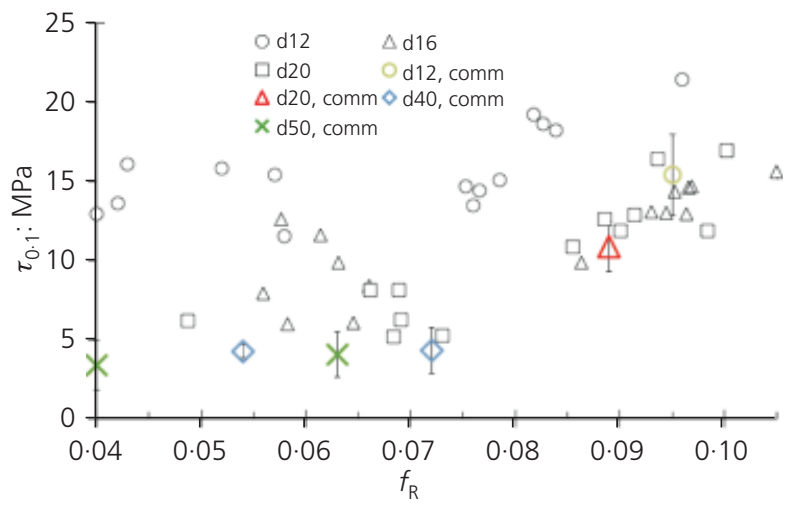

Figure 11. Influence of relative rib area on the bond stiffness in NSC: bond stress $\tau_{0.1}$ measured at unloaded-end slip $\left(\delta_{\mathrm{u}}\right)$ of $0.1 \mathrm{~mm}$ plotted against relative rib area $\left(f_{\mathrm{R}}\right)$. Notation $\mathrm{d} 12, \mathrm{~d} 16$, etc. refers to bar diameter, in $\mathrm{mm}$ 


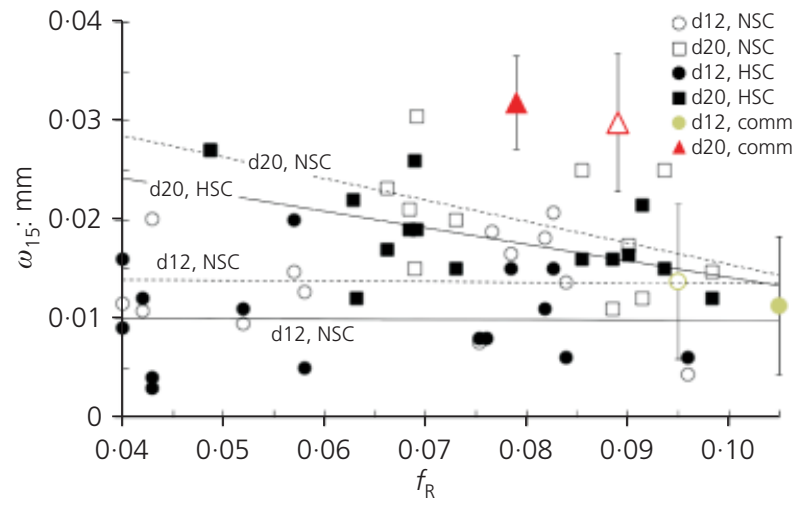

Figure 12. Transverse deformation $\left(w_{15}\right)$ of the specimens plotted against bond index $\left(f_{\mathrm{R}}\right)$, at the bond stress of $15 \mathrm{MPa}$. Notation $\mathrm{d} 12$, d16, etc. refers to bar diameter, in $\mathrm{mm}$; comm represents commercial hot-rolled bar

results, Figure 12 shows a clear and sizeable dependence of the splitting crack on the relative rib area, both in NSC and in HSC specimens. The transverse deformation $w_{15}$ shows an upward trend with bar dimension and a downward trend with an increase in bond index.

The experimental results confirm the analytical studies of Cairns and Jones (1995b), who suggested a lower wedging action of an anchored bar with a higher relative rib area; this allows a higher bond strength to be achieved, as clearly shown in Figures 5, 7 and 8. Furthermore, it is worth pointing out the significant influence of bar dimension on bursting force (size effect), especially in rebars with a low bond index. By assuming that the measured transverse deformation is proportional to the radial force exerted by ribs, the test results indicate that the bursting action generated by $20 \mathrm{~mm}$ diameter bars is twice that generated by $12 \mathrm{~mm}$ diameter bars, both in NSC and in HSC for a bond index of 0.05 ; this difference reduces to $10 \%$ for a bond index of $0 \cdot 10$. Although it is difficult to accurately quantify the difference between the bursting force in HSC and NSC because of the wide scatter of the test results, the linear regressions in Figure 12 show the tendency of bond to exert a greater wedging action in NSC (dashed lines) rather than in HSC (continuous lines) for a given bar diameter. This is because of the lower porosity of HSC, which influences the local behaviour around the ribs of the rebars.

\section{Concluding remarks}

This paper has discussed an experimental programme of 151 pull-out tests carried out to better understand the influence of the relative rib area of reinforcing bars on bond. The experimental results provide information on bond strength, stiffness, size effect and wedge action generated by ribs.

The experiments were carried out on 12, 16 and $20 \mathrm{~mm}$ diameter machined bars of high steel grade, having a bond index ranging from 0.04 to $0 \cdot 105$, as well as on $12,20,40$ and $50 \mathrm{~mm}$ diameter commercial hot-rolled bars with a bond index greater than $0 \cdot 063$. The 40 and $50 \mathrm{~mm}$ diameter bars were also tested with a bond index lower than the minimum value required by Eurocode 2. In the pull-out tests, all the rebars were embedded for a length of five times the bar diameter $d$ in cubic specimens (without transverse reinforcement), with a cover of $4 \cdot 5 d$.

The following conclusions can be drawn from the test results.

(a) Bond strength is strongly dependent on the relative rib area. For example, an increase in bond index from 0.04 to $0 \cdot 10$ leads to an increase of bond strength of up to $40 \%$. This enhancement may be due to the lower wedging action generated by highly ribbed bars, which reduces the risk of splitting failure.

(b) The minimum bond strength, as determined from the specimens with a lower bond index, is significantly higher than the design value required by MC2010; this underlines the possibility of reducing the minimum value of relative rib area prescribed by codes without impairing the safety requirement of bond strength. This would enhance the structural ductility of RC members because of the greater strain penetration allowed by the increase in bond slip, especially if high-performance concrete is used.

(c) On the other hand, the tests results indicate that the adoption of a high bond index can limit the longitudinal splitting crack width, due to a reduced wedging action of the bar ribs.

(d) Finally, the test results confirm that bond behaviour is affected by a size effect, both in terms of strength and stiffness: for a bar diameter increasing from 12 to $50 \mathrm{~mm}$, the reduction in bond strength is about $25 \%$ while the reduction in secant bond stiffness is greater than $70 \%$.

\section{Acknowledgements}

The authors gratefully acknowledge the support of Leali S.p.A (Odolo, Italy) for financing a research project on large-diameter bars. Special acknowledgment goes to engineers A. Moreschi, M. Galuppi, M. Ferri and M. Cristini for their help in carrying out the tests. Finally, the authors thank technicians A. Delbarba and A. Coffetti, of the Laboratory P. Pisa of the University of Brescia, for their technical support. 


\section{Appendix}

The geometric properties of the machined bars are shown in Table 8 .

\begin{tabular}{|c|c|c|c|c|}
\hline Bar No. & $d_{\mathrm{e}}: \mathrm{mm}$ & a: $\mathrm{mm}$ & $\mathrm{s}: \mathrm{mm}$ & $f_{\mathrm{R}}$ \\
\hline \multicolumn{5}{|c|}{ Series 1 and $7, f_{\mathrm{Rm}}=0.047$} \\
\hline 1,49 & $13 \cdot 0$ & $0 \cdot 349$ & $8 \cdot 75$ & 0.043 \\
\hline 2,50 & $13 \cdot 0$ & 0.469 & $8 \cdot 75$ & 0.058 \\
\hline 3,51 & $13 \cdot 0$ & 0.337 & 8.75 & 0.042 \\
\hline 4,52 & $13 \cdot 0$ & 0.466 & 8.75 & 0.057 \\
\hline 5,53 & $13 \cdot 0$ & $0 \cdot 326$ & 8.75 & 0.040 \\
\hline 6,54 & $13 \cdot 0$ & 0.418 & $8 \cdot 75$ & 0.052 \\
\hline 7,55 & $13 \cdot 0$ & $0 \cdot 344$ & $8 \cdot 75$ & 0.043 \\
\hline 8,56 & $13 \cdot 0$ & $0 \cdot 321$ & 8.75 & 0.040 \\
\hline \multicolumn{5}{|c|}{ Series 2 and $8, f_{\mathrm{Rm}}=0.061$} \\
\hline 9,57 & $17 \cdot 0$ & 0.620 & $10 \cdot 95$ & 0.058 \\
\hline 10,58 & $17 \cdot 1$ & 0.686 & $10 \cdot 95$ & 0.065 \\
\hline 11,59 & $17 \cdot 0$ & 0.594 & $10 \cdot 95$ & 0.056 \\
\hline 12,60 & $17 \cdot 0$ & 0.656 & $10 \cdot 95$ & 0.061 \\
\hline 13,61 & $17 \cdot 0$ & 0.623 & $10 \cdot 95$ & 0.059 \\
\hline 14,62 & $17 \cdot 1$ & 0.671 & $10 \cdot 95$ & 0.063 \\
\hline 15,63 & $17 \cdot 1$ & 0.610 & $10 \cdot 95$ & 0.058 \\
\hline 16,64 & $17 \cdot 1$ & 0.703 & $10 \cdot 95$ & 0.066 \\
\hline \multicolumn{5}{|c|}{ Series 3 and $9, f_{\mathrm{Rm}}=0.065$} \\
\hline 17,65 & $21 \cdot 0$ & 0.879 & $13 \cdot 90$ & 0.063 \\
\hline 18,66 & $21 \cdot 0$ & 1.023 & $13 \cdot 90$ & 0.073 \\
\hline 19,67 & $21 \cdot 0$ & 0.955 & $13 \cdot 90$ & 0.068 \\
\hline 20,68 & $21 \cdot 0$ & 0.671 & $13 \cdot 90$ & 0.049 \\
\hline 21,69 & $21 \cdot 0$ & 0.923 & $13 \cdot 90$ & 0.066 \\
\hline 22,70 & $21 \cdot 0$ & 0.966 & $13 \cdot 90$ & 0.069 \\
\hline 23,71 & $21 \cdot 0$ & 0.869 & $13 \cdot 90$ & 0.063 \\
\hline 24,72 & $21 \cdot 0$ & 0.963 & $13 \cdot 90$ & 0.069 \\
\hline \multicolumn{5}{|c|}{ Series 4 and $10, f_{\mathrm{Rm}}=0.081$} \\
\hline 25,73 & $13 \cdot 0$ & 0.438 & $6 \cdot 00$ & 0.076 \\
\hline 26,74 & $13 \cdot 0$ & 0.470 & $6 \cdot 00$ & 0.084 \\
\hline 27,75 & $13 \cdot 0$ & 0.463 & $6 \cdot 00$ & 0.083 \\
\hline 28,76 & $13 \cdot 0$ & 0.458 & $6 \cdot 00$ & 0.082 \\
\hline 29,77 & $13 \cdot 0$ & 0.439 & $6 \cdot 00$ & 0.077 \\
\hline 30,78 & $13 \cdot 0$ & 0.431 & $6 \cdot 00$ & 0.075 \\
\hline 31,79 & $13 \cdot 0$ & 0.550 & $6 \cdot 00$ & 0.096 \\
\hline 32,80 & $13 \cdot 0$ & 0.450 & $6 \cdot 00$ & 0.079 \\
\hline \multicolumn{5}{|c|}{ Series 5 and $11, f_{\mathrm{Rm}}=0.095$} \\
\hline 33,81 & $17 \cdot 0$ & 0.628 & $7 \cdot 50$ & 0.086 \\
\hline 34,82 & $17 \cdot 1$ & 0.694 & $7 \cdot 50$ & 0.095 \\
\hline 35,83 & $17 \cdot 1$ & 0.707 & $7 \cdot 50$ & 0.097 \\
\hline 36,84 & $17 \cdot 2$ & 0.698 & $7 \cdot 50$ & 0.096 \\
\hline 37,85 & $17 \cdot 3$ & 0.755 & $7 \cdot 50$ & $0 \cdot 106$ \\
\hline 38,86 & $17 \cdot 0$ & 0.685 & $7 \cdot 50$ & 0.094 \\
\hline 39,87 & $17 \cdot 0$ & 0.711 & $7 \cdot 50$ & 0.097 \\
\hline 40,88 & $17 \cdot 1$ & 0.679 & $7 \cdot 50$ & 0.093 \\
\hline
\end{tabular}

Series 6 and $12, f_{\mathrm{Rm}}=0.092$

$\begin{array}{lllll}41,89 & 20.9 & 0.836 & 9.50 & 0.089 \\ 42,90 & 21.0 & 0.949 & 9.50 & 0.100 \\ 43,91 & 20.9 & 0.808 & 9.50 & 0.086 \\ 44,92 & 21.1 & 0.880 & 9.50 & 0.094 \\ 45,93 & 21.0 & 0.850 & 9.50 & 0.090 \\ 46,94 & 21.1 & 0.927 & 9.50 & 0.098 \\ 47,95 & 20.9 & 0.867 & 9.50 & 0.091 \\ 48,96 & 21.0 & 0.862 & 9.50 & 0.091\end{array}$

Table 8. Geometric properties of machined bars

\section{REFERENCES}

Bamonte PF and Gambarova PG (2007) High-bond in NSC and HSC: a study on size effect and on the local bond stress-slip law. Journal of Structural Engineering, ASCE 133(2): 225234.

Bazant ZP and Sener S (1988) Size effect in pull-out tests. $A C I$ Materials Journal 85(5): 347-351.

Cairns J and Abdullah R (1995) An evaluation of bond pullout tests and their relevance to structural performance. The Structural Engineer 73(1): 179-185.

Cairns J and Jones K (1995a) The splitting forces generated by bond. Magazine of Concrete Research 47(171): 153-165.

Cairns J and Jones K (1995b) Influence of rib geometry on strength of lapped joints: an experimental and analytical study. Magazine of Concrete Research 47(172): 253-262.

Cairns J and Plizzari GA (2003) Towards a harmonised European bond test. Material and Structures 36(262): 498-506.

CEN (European Committee for Standardisation) (2004) Eurocode 2: EN 1992-1-1: Design of concrete structures. Part 1-1: General rules and rules for buildings. CEN, Brussels, Belgium.

Dancygier A and Katz A (2010) Bond between deformed reinforcement and normal and high-strength concrete with and without fibers. Materials and Structures 43(2): 839-856.

Darwin D and Graham EK (1993) Effect of deformation height and spacing on bond strength of reinforcing bars. $A C I$ Structural Journal 90(6): 646-657.

Darwin D, McCabe SL, Idun EK and Schoenekase SP (1992) Development length criteria: bars not confined by transverse reinforcement. ACI Structural Journal 89(6): 709-720.

Eligehausen R and Mayer U (2000) Investigation on the Influence of the Relative Rib Area of Reinforcement on the Structural Behaviour of Reinforced Members in the Serviceability and Ultimate Limit State. Deutscher Ausschuss für Stahlbeton, Beuth, Berlin, Germany (in German).

Eligehausen R, Popov EP and Bertero VV (1983) Local Bond Stress-slip Relationships of Deformed Bars Under Generalized Excitations. University of California, Berkeley, CA, USA, Report UCB/EERC 83-23. 
fib (International Federation for Structural Concrete) (2013) Model Code for Concrete Structures 2010. Ernst \& Sohn, Berlin, Germany.

fib (2014) Task Group 4.5: Bond Models, Convenor J. Cairns. Bond and Anchorage of Reinforcement - Background to Revisions in the fib Model Code 2010 - final draft, in press. fib, Lausanne, Switzerland.

Gambarova PG and Rosati GP (1997) Bond and splitting in bar pull-out: behavioural laws and concrete-cover role. Magazine of Concrete Research 48(6): 99-110.

Gambarova PG, Plizzari GA, Balazs GL et al. (2000) Bond mechanics including pull-out and splitting failures. In $f i b$ Bulletin 10: Bond of Reinforcement in Concrete. fib, Lausanne, Switzerland, pp. 1-98.

Giuriani E (1982) On the effective axial stiffness of a bar in cracked concrete. In Bond in Concrete (Bartos P (ed.)). Applied Science, London, UK, pp. 107-126.

Giuriani E and Plizzari GA (1998) Interrelation of splitting and flexural cracks in R. C. beams. Journal of Structural Engineering, ASCE 124(9): 1032-1040.

Giuriani E, Plizzari GA and Schumm C (1991) Role of stirrups and residual tensile strength of cracker concrete on bond. Journal of Structural Engineering, ASCE 117(1): 1-18.

Harajli MH, Hout M and Jalkh W (1995) Local bond stress-slip behaviour of reinforcing bars embedded in plain and fiber concrete. ACI Materials Journal 92(4): 343-354.

Ichinose T, Kanayama Y, Inoue $Y$ and Bolander JE (2004) Size effect on bond strength of deformed bars. Construction and Building Materials 18(7): 549-558.

ISO (International Organization for Standardization) (2010) EN 15630-1: Steel for the reinforcement and prestressing of concrete - Test methods - Part 1: Reinforcing bars, rod and wire. ISO, Geneva, Switzerland.

Jansson A, Lofgren I, Lundgren K and Gylltoft K (2012) Bond of reinforcement in self-compacting steel-fibre-reinforced concrete. Magazine of Concrete Research 64(7): 617-630.
Metelli G, Cominoli L, Panteghini A and Plizzari GA (2010) Numerical and Experimental Study on the Use of Large Diameter Ribbed Bars in Concrete Structures. DICATA, University of Brescia, Brescia, Italy, Technical report 11 (in Italian).

Plizzari GA, Deldossi MA and Massimo S (1998) Transverse reinforcement effects on anchored deformed bars. Magazine of Concrete Research 50(2): 161-177.

Reinhardt HW and van der Veen C (1990) Applications of fracture mechanics to reinforced concrete. In Splitting Failure of a Strain-softening Material Due to Bond Stresses (Carpinteri A (ed.)). Elsevier, Barking, UK, pp. 333-346.

Rhem G (1969) Evaluation Criteria for High-bond Rebars. Festschrift Rüsch. Ernst and Sohn, Berlin, (in German).

Rilem/CEB/FIP (1978) Bond Test for Reinforcing Steel 2: Pull-out Test. Recommendation RC6. Rilem, Bagneux, France.

Tepfers R (1973) A Theory of Bond Applied to Overlapped Tensile Reinforcement Splices for Deformed Bars. Chalmers University of Technology, Goteborg, Sweden, Publication $73 / 2$.

Walker PR, Batayneh MK and Regan PE (1999) Measured and design bond strengths of deformed bars, including the effect of lateral compression. Magazine of Concrete Research 51(1): $13-26$.

Wildermuth A and Hofmann J (2012) Effect of the bond behaviour of rebars and its evaluation by simplified test specimens. Proceedings of the 4th International Conference on Bond in Concrete, Brescia, Italy, 1, 75-80.

Xu F, Wu ZM, Zheng JJ, Hu Y and Li QB (2011) Experimental study on the bond behavior of reinforcing bars embedded in concrete subjected to lateral pressure. Journal of Materials in Civil Engineering 24(1): 125-133.

Zuo J and Darwin D (2000) Bond slip of high relative rib area under cycling loading. ACI Structural Journal 97(2): 331335 .

\section{WHAT DO YOU THINK?}

To discuss this paper, please submit up to 500 words to the editor at journals@ice.org.uk. Your contribution will be forwarded to the author(s) for a reply and, if considered appropriate by the editorial panel, will be published as a discussion in a future issue of the journal. 